\title{
C9orf72-associated SMCR8 protein binds in the ubiquitin pathway and with proteins linked with neurological disease
}

John L. Goodier ${ }^{1 *}$, Alisha O. Soares', Gavin C. Pereira', Lauren R. DeVine², Laura Sanchez ${ }^{3}$, Robert N. Cole ${ }^{2}$ and Jose Luis García-Pérez ${ }^{3,4}$

\begin{abstract}
A pathogenic GGGCCC hexanucleotide expansion in the first intron/promoter region of the C9orf72 gene is the most common mutation associated with amyotrophic lateral sclerosis (ALS). The C9orf72 gene product forms a complex with SMCR8 (Smith-Magenis Syndrome Chromosome Region, Candidate 8) and WDR41 (WD Repeat domain 41) proteins. Recent studies have indicated roles for the complex in autophagy regulation, vesicle trafficking, and immune response in transgenic mice, however a direct connection with ALS etiology remains unclear. With the aim of increasing understanding of the multi-functional C9orf72-SMCR8-WDR41 complex, we determined by mass spectrometry analysis the proteins that directly associate with SMCR8. SMCR8 protein binds many components of the ubiquitin-proteasome system, and we demonstrate its poly-ubiquitination without obvious degradation. Evidence is also presented for localization of endogenous SMCR8 protein to cytoplasmic stress granules. However, in several cell lines we failed to reproduce previous observations that C9orf72 protein enters these granules. SMCR8 protein associates with many products of genes associated with various Mendelian neurological disorders in addition to ALS, implicating SMCR8-containing complexes in a range of neuropathologies. We reinforce previous observations that SMCR8 and C9orf72 protein levels are positively linked, and now show in vivo that SMCR8 protein levels are greatly reduced in brain tissues of C9orf72 gene expansion carrier individuals. While further study is required, these data suggest that SMCR8 protein level might prove a useful biomarker for the C9orf72 expansion in ALS.
\end{abstract}

Keywords: Amyotrophic lateral sclerosis, Autophagy, Biomarker, Mass spectrometry, Proteasome, Stress granules, Ubiquitin

\section{Introduction}

Amyotrophic lateral sclerosis (ALS) is a fatal neurodegenerative disease that afflicts about 1 in 50,000 people each year and involves loss of upper and lower motor neurons [1]. Death typically follows 2 to 3 years after first onset. About $95 \%$ of cases are sporadic, while the rest have a family history of the disease. ALS also has

\footnotetext{
* Correspondence: jgoodier@jhmi.edu

1 McKusick-Nathans Department of Genetic Medicine, Johns Hopkins University School of Medicine, Baltimore, MD, USA

Full list of author information is available at the end of the article
}

overlapping clinical presentations with frontotemporal lobar degeneration (FTLD) and its most common subtype frontotemporal dementia (FTD), a neurological condition affecting the frontal and temporal lobes and marked by cognitive and behavioral impairment [2]. About 20\% of ALS patients also exhibit FTLD, and ALS and FTLD have been considered to be part of a continuous disease spectrum [3].

A pathogenic GGGCCC hexanucleotide expansion in the first intron/promoter region of the C9orf72 gene is the most common mutation associated with both ALS 
( $11 \%$ of all cases) and FTLD/FTD ( 13\%) [3-6]. Three possible non-exclusive mechanisms have been proposed by which the C9orf72 repeat expansion may cause ALSFTD: 1) haploinsufficiency and loss of C9orf72 protein function, 2) repeat-associated non-AUG (RAN) translation of the hexanucleotide repeats generating dipeptide repeats that aggregate in toxic neuronal cytoplasmic and nuclear aggregates, and 3) toxic gain-of-function from repeat-containing RNA which forms nuclear foci that sequester hexanucleotode repeat-binding proteins (reviewed in [7-10]). While most studies have focused on increasedtoxicity, accumulating evidence argues that a loss-of-function mechanism may also contribute to neurodegeneration. Consistently, various studies have reported a reduction in C9orf $72 \mathrm{mRNA}$ and/or protein expression in brain and induced pluripotent stem cell (iPSC)-derived neuronal lines of some C9orf72 ALS (C9ALS) and FTD patients [4-6, 11-25].

A series of studies have shown that the long isoform of human C9orf72 protein forms a complex with SMCR8 (Smith-Magenis Syndrome Chromosome Region, Candidate 8) and WDR41 (WD Repeat domain 41) proteins [22, 26-35]. The SMCR8 gene is within the deleted region of chromosome 17 associated with SmithMagenis Syndrome (SMS), a developmental disorder of children involving intellectual disability, distinctive facial features, and behavioral problems, but no reported motor defects $[36,37]$. WDR41 is a member of the WDrepeat family of proteins that act as protein-protein or protein-DNA interaction scaffolds for a variety of cellular functions [38]. SNPs within the WDR41 gene region have been associated with human caudate volume [39].

Bioinformatic analyses first identified both C9orf72 and SMCR8 proteins as having DENN (Differentially Expressed in Normal and Neoplastic cells) domains that are present in guanine nucleotide exchange factors (GEFs) for Rabs, multi-functional small GTPases involved in intracellular membrane trafficking and fusion, vesicle formation and transport, and autophagy [40-42]. The autolysomal-autophagy pathway involves generation of the autophagosome, an organelle surrounded by a double lipid bilayer. Autophagosomes engulf cytoplasmic components, such as protein aggregates, damaged organelles, and foreign pathogens, and fuse with lysosomes to generate autolysosomes that mediate degradation of the cargo. Autophagosomes also fuse with endosomes, forming an intermediate organelle called the amphisome, before fusion with lysosomes. Various studies have linked wild-type C9orf72 protein with proteostasis, showing that, in complex with SMCR8 and WDR41, it bind Rabs and plays roles in autophagy and initiation of autophagosome formation, as well as being linked by function and colocalization to endocytosis and lysosomal and endosomal trafficking $([9,21-23,26,28,29,31,32,34$,
43-48], and Discussion for review). A role in the endolysosome pathway has also been shown for the C. elegans C9orf72 ortholog alfa-1 [49]. Aoki et al. [46] linked the interaction of C9orf72 and RAB7L1 with regulation of vesicle trafficking, and WDR41 is necessary for recruitment of the C9orf72 complex to lysosomes [35, 50]. Thus, C9orf72 is a regulator of cellular proteostasis.

Additional roles for the C9orf72 complex have also been reported. C9orf72 alters phosporylation of cofilin and activates the small GTPase ADP-ribosylation factor$1 / 2($ ARF1/2) involved in actin dynamics [51]. Altered C9orf72 protein levels also causes changes in glutamatergic receptor levels, glutamate cycling and endothelin signaling, and excitotoxicity in response to glutamate, as well as widespread transcriptional changes [21, 5254]. However, the consequences of loss of C9orf72 protein for motor neuron function remain unclear. In vivo, diminished motor function and axonal degeneration of motor neurons have been reported in zebrafish and $C$. elegans depleted of C9orf72 [55, 56]. However, subsequent studies detected no or only mild motor function defects in mice deficient for murine C9orf72 ortholog $3110043 \mathrm{O} 21$ Rik [45]. On the other hand, in a gain-offunction C9ALS/FTD mouse model, Shao et al. [57] found that $3110043 \mathrm{O} 21$ Rik haploinsufficiency or loss was associated with increased motor behavior deficits in a dose-dependent manner, while Liang et al. [25] reported that Smcr8 knockout (KO) mice displayed motor behavior defects and axonal swelling. While effects on motor function are uncertain, immune system pathology, spleen and lymph node enlargement, defects in macrophage, myeloid and microglial cell function, altered lysosomal trafficking, and decreased body weight and survival have all been reported for C9orf72 or SMCR8 knockout mice [21, 28, 32, 45, 58-65]. Despite these findings, so far no pathogenic loss-of-function coding mutation in C9orf72, SMCR8 or WDR41 genes has been found [66].

To increase understanding of the diverse functions of the C9orf72-SMCR8-WDR41 complex, we sought to determine by mass spectroscopy (MS) analyses the interactome composition of the SMCR8 component. Notably, we found that the SMCR8 complex includes numerous ubiquitin-related proteins and products of genes associated with numerous Mendelian neurological disorders. MS analyses, co-IP experiments, and association of SMCR8 with cytoplasmic stress granules (SGs) in cultured cells support a link between SMCR8 and the ubiquitin pathway. Furthermore, we reinforce previous observations that SMCR8 and C9orf72 protein levels are positively linked, now showing in vivo that SMCR8 might prove to be a useful biomarker for the C9orf72 expansion mutation in ALS patients. 


\section{Materials and methods}

\section{Plasmid constructs}

Ultimate ORF cDNA clones (Invitrogen), with V5epitope tags and tobacco etch virus (TEV) protease cleavage sites on their N-termini, were recloned by shuttling them from pENTR221 vector into pcDNA3.1/nV5DEST using Gateway Technology (Invitrogen). Ultimate ORF Clones included AIFM1 (clone identifier IOH61019), BAG5 (IOH26366), C9orf72 (IOH45695), DARS (IOH4209), DCTN1 (IOH42830), DDB1 (IOH13816), DNAJC7 (IOH14566), FKBP5 (IOH13816), G3BP1 (IOH7337), GTF2I (IOH62625), HSPB1 (IOH10530), MAGED2 (IOH3451), PARK7 (IOH3149), PPP2R1A (IOH13670), PSMA7 (IOH5011), PSMC5 (IOH3508), PSMD14 (IOH40112), QARS (IOH3529), RAB1A (IOH2921), RAB7A (IOH40569), RAB11A (IOH13764), RANGAP1 (IOH13287), $\quad$ RO60 (IOH22411), RNF40 (IOH6599), RUVBL2 (IOH3426), SLC25A6 (IOH5815), SQSTM1 (IOH5103), STIP1 (IOH5061), STUB1 (IOH56981), SUGT1 (IOH2955), and TBK1 (IOH21006).

Clones purchased or obtained as gifts included FLAGUBR5 (pCMV-Tag2B EDD; Addgene plasmid \#37188 [67]), pcDNA5 FRT/TO FLAG-SMCR8 (FL-SMCR8), pcDNA5 FRT/TO HA-SMCR8, and pcDNA5 FRT/TO GFP SMCR8 (MRC PPU Reagents and Services, University of Dundee), GFP-(GA) ${ }_{50}$ (L. Petrucelli, Mayo Clinic, Florida, [68]) pRK5-myc-TDP-43 (J. Wang, Johns Hopkins University, [69]), mRFP-UBB (Addgene plasmid \#11935, [70]), HA-UBB (V. Dawson, Johns Hopkins University, [71]), and pDest51-USP9X-V5 (R. Hughes, Buck Institute for Research on Aging, [72]). FL-UBB was generated using Ultimate ORF Clone IOH56688 and modified Gateway vector pEZYflag (Addgene plasmid \#18700, Y.-Z. Zhang). C9orf72-FL, RO60-FL, SMCR8-V5, and WDR41-FL constructs were generated by PCRamplification of Ultimate ORF cDNA clones, using a primer with AAG linker and C-terminal FLAG- or V5-tag, and cloning of the products in pcDNA6/myc-His $B$ (pcDNA6, Invitrogen).

\section{Cell line and tissue samples}

Human embryonal carcinoma 2102Ep (a gift from P.K. Andrews, University of Sheffield, UK) and nTERA2D1 cells, human cervical cancer HeLa-JVM cells [73], human embryonic kidney (HEK) 293T cells (ATCC), human neuroblastoma SH-SY-5Y cells (ATCC CRL-2266), and mouse neuroblastoma Neuro2A cells (a gift from D. Hackam, JHU) were grown in Dulbecco's modified Eagle's Medium. Mouse hybrid spinal cord neuron/neuroblastoma NSC-34 cells (a gift from D. Griffen, JHU) and human neuroblastoma SK-N-SH cells (a parental line of SH-SY-5Y cells; gifts of D. Valle, JHU) were grown in Eagle's Minimum Essential Medium. Medium was supplemented with 10\% FBS (Hyclone), GlutaMax, and Pen-Strep (Invitrogen). Plasmid transfections used FuGENE HD (Promega) reagent. As necessary, cells were treated with $20 \mu \mathrm{M}$ MG-132 proteasome inhibitor (Cell Signaling) for about $20 \mathrm{~h}$ to inhibit protein degradation, or with $0.25 \mathrm{mM}$ sodium arsenite $\left(\mathrm{NaAsO}_{2}\right)$ or 3 $\mathrm{mM}$ dithiothreitol (DTT, Sigma) for $80 \mathrm{~min}$ and $2 \mathrm{~h}$, respectively, to induce cytoplasmic SGs.

Post-mortem ALS spinal cord and unaffected control brain motor cortex tissues were obtained from Drs. J. Ravits and R. Batra of the Department of Neurosciences, University of California San Diego School of Medicine [74], and C9ALS and unaffected control samples were from the Target ALS Multicenter Postmortem Tissue Core (Table S4). C9ALS samples had been confirmed for the C9orf72 expansion using repeat-primed PCR (RP-PCR) and Illumina Expansion Hunter (M. Harms, Columbia University, pers. comm.). Frozen spinal cord tissues were obtained from the University of Maryland Brain and Tissue Bank of the NIH NeuroBioBank. Frozen C9ALS and unaffected control cerebrospinal fluid (CSF) samples were from the Northeast ALS Consortium (NEALS). CSF was resuspended directly in 3X SDS loading buffer or first concentrated by tricholoracetic acid precipitation, and then analyzed by Western blotting using $\alpha$-SMCR8 antibodies. Up to $20 \mu \mathrm{g}$ of CSF total protein were loaded per well.

\section{Protein isolation and immunoprecipitation}

For MS sequence determination, HEK 293T cells in $\mathrm{T}_{75}$ flasks were transfected using FuGENE HD (Promega) with $15 \mu \mathrm{g}$ of FL-SMCR8, C9orf72-FL, or pcDNA6/mycHis B empty vector and expanded for approximately 45 $h$, followed by whole cell lysate preparation by sonication using a Diagenode Bioruptor. IP and sample recovery were as previously described $[75,76]$. Treatment of samples with $25 \mu \mathrm{g} / \mathrm{ml}$ DNase-free RNase (Roche) and $25 \mu \mathrm{g} / \mathrm{ml}$ RNaseA (Qiagen) was conducted in the absence of RNase inhibitors.

For other protein extracts, tissues or cells were lysed in RIPA buffer (Sigma) with Mammalian Protease Inhibitor Cocktail and phenylmethanesulfonyl fluoride (Sigma) and homogenized by Diagenode Bioruptor. For tissues, $2 \mathrm{~mm}$ ziconium silicate beads (Next Advance, Inc.) were added to the tubes. Supernatants were recovered by centrifugation at $11 \mathrm{~K} \mathrm{rpm}$ at $4{ }^{\circ} \mathrm{C}$ for $15 \mathrm{~min}$ and resuspended in 3X SDS loading buffer.

For each interaction co-IP (Fig. 2), extracts from approximately $5 \times 10^{6} 293 \mathrm{~T}$ cells in $\mathrm{T}_{75}$ flasks transfected with tagged SMCR8 and test protein constructs were prepared in $700 \mu \mathrm{l}$ of lysis buffer $(160 \mathrm{mM} \mathrm{NaCl}, 50 \mathrm{mM}$ Tris, $1 \mathrm{mM}$ EDTA, 0.25\% NP40) containing protease and phosphatase inhibitors (Sigma), and RNasin (Qiagen) and vanadyl ribonucleoside complexes (Sigma) as 
required, and immunoprecipitated as previously described $[75,76]$.

\section{Antibody analyses}

Commercial antibodies included mouse (ms) $\alpha-\mathrm{V} 5$-tag (Invitrogen), rabbit (rb) $\alpha$-DYKDDDDK-tag ( $\alpha$-FLAG) (D6W5B), ms $\alpha$-HA-tag (6E2), rb $\alpha$-Myc-tag (71D10), rb $\alpha$-HSP90, and ms $\alpha$-Ubiquitin (UBB) (P4D1) (Cell Signaling Technology), rb $\alpha-4 \mathrm{E}-\mathrm{T}, \mathrm{rb} \alpha-\mathrm{C} 9$ orf72 (S-14), goat (gt) $\alpha$-eIF3n (N-20), ms $\alpha$-p70 S6 kinase (which recognizes HEDLS/EDC4 [77]), gt $\alpha$-TIA1 (C-20), and rb $\alpha$ WDR41 (S-12) (Santa Cruz Biotechnology), rb $\alpha$-SMCR8 (ab186504, epitope region 841-890) and $\mathrm{rb} \alpha$-SMCR8 (ab202283, epitope region 600-650) (Abcam), rb $\alpha$ C9orf72 (22637-1-AP), rb $\alpha$-SMCR8 (21125-1-AP, epitope region 588-937), and rb $\alpha$-WDR41 (26817-1-AP) (Proteintech), and rb $\alpha$-SMCR8 (A304-694A, epitope region 600-650) (Bethyl). Monoclonal ms $\alpha-4$ H1-ORF1 was kindly provided by Dr. K. Burns (JHU, USA, also Millipore MABC1152, [78]), rb $\alpha-C 9-\mathrm{L}$ and $\mathrm{rb} \alpha-\mathrm{C} 9-\mathrm{S}$ antibodies by Dr. J. Robertson (U. Toronto, Canada, [19, 79]), and $\alpha-G W 182$ human serum (IC-6) by Dr. M. Fritzler (U. Calgary, [80]). Donkey Cy3-, DyLight 488-, or DyLight 549-conjugated, and HRP-conjugated secondary antibodies were from Jackson ImmunoResearch Laboratories.

Western blotting, immunofluorescence (IF) microscopy, and fluorescent in situ hybridization (FISH) were performed as described [81, 82]. All Western blotting used NuPAGE $4-12 \%$ Bis-Tris gels and MOPS buffer, except brain tissue analyses of Fig. 5b,c which used NuPAGE 3-8\% Tris-acetate gels (Thermo Fisher). Detection used Supersignal West Pico or West Pico Plus Chemiluminescent Substrate (Thermo Fisher) with ECL Hyperfilm (MilliporeSigma).

Immunostained cells were examined using a Nikon Eclipse Ti-A1 confocal microscope with NIS-Elements AR software.

\section{MS sequencing and data analyses}

MS sequencing and database analyses was performed by the Johns Hopkins Mass Spectrometry and Proteomics Facility as previously described $[75,76]$. Peptide sequences were identified using Proteome Discoverer and Mascot software (Matrix Science) to search the NCBInr 167 database, including gly-gly modifiation on lysine as a variable protein modification. False discovery rate (FDR) was set at 1.0. Mascot search result *.dat files were processed in Scaffold (Proteome Software, Inc.) to validate protein and peptide identifications. Exclusion criteria for proteins are described in the Results section.

Functional analyses of gene lists obtained by MS sequencing utilized DAVID v6.7 (Database for Annotation, Visualization and Integrated Discovery [83]). Protein multiple alignments used Clustal Omega 1.2.1 (EMBLEBI) and the ESPript 3.0 web sever (http://espript.ibcp. fr/ESPript/ESPript/). RNA-Seq datasets from public databases (GEO GSE67196 and NeuroLINCS dbGaP Study phs001231 SRP098831) were analyzed for gene expression using the software package TETranscripts as previously described $[84,85]$. Ubiquitination prediction algorithms included UbPred (http://www.ubpred.org, [86]), BDM-PUB (http://bdmpub.biocuckoo.org), and UbiSite (http://csb.cse.yzu.edu.tw/UbiSite/).

\section{Results}

Confirmation of the SMCR8-C9orf72-WDR41 complex and detection using commercial antibodies

Previous studies characterizing the C9orf72 proteome identified a complex consisting of the proteins C9orf72, SMCR8 and WDR41 [22, 26-35]. By means of coimmunoprecipitation (co-IP) and MS sequencing, here we sought to identify proteins that associate with SMCR8. First, we confirmed that C9orf72, SMCR8 and WDR41 proteins formed a stable complex in our overexpression experimental system using human embryonic kidney (HEK) 293T cells. Consistent with previous reports, even in the presence of RNase, epitope-tagged SMCR8 and C9orf72 co-IPed C9orf72 and SMCR8 proteins, respectively (Fig. 1a, b). Furthermore, we confirmed that FLAG-tagged WDR41 interacts with endogenous C9orf72 and SMCR8 (Fig. 1c).

We next assessed the efficacy of several commercial antibodies against these proteins. Human C9orf72 expresses a 54-kilodalton $(\mathrm{kD})$ long protein isoform (C9-L) and a $25-\mathrm{kD}$ short isoform (C9-S). It has been noted that commercial C9orf72 antibodies often detect additional bands other than C9-L or fail to detect C9-S [17, 19, 23]. Consistent with this observation, the Santa Cruz S-14 antibody ( $\alpha$-C9orf72-SC) detected multiple bands in whole cell lysates as well as products of size consistent with both endogenous $\mathrm{C} 9-\mathrm{L}$ and $\mathrm{C} 9-\mathrm{S}$; only $\mathrm{C} 9-\mathrm{L}$ coIPed with tagged SMCR8 or WDR41 (Fig. 1a,c). Similarly, in both cultured cells and brain and spinal cord tissue lysates, the Proteintech 22,637-1-AP antibody ( $\alpha$ C9orf72-PT) marked a major band consistent with C9-L (arrow), plus additional products (Fig. S1A). A small number of non-commercial C9orf72-specific antibodies have also been described [17, 23, 30,79].

Both mouse and human SMCR8 have two predicted isoforms, the full length $105-\mathrm{kD}$ protein and a Cterminal truncated $87.4-\mathrm{kD}$ isoform generated by alternate splicing [36]. Our search of GenBank revealed additional human SMRC8 mRNA isoforms potentially encoding 35.9-kD (accession numbers BC001018, BC005067), 75.2-kD (AK296847.1), and 93-kD (BC101116, BC101117) protein products. Tissue-specific transcripts of various sizes have also been experimentally 


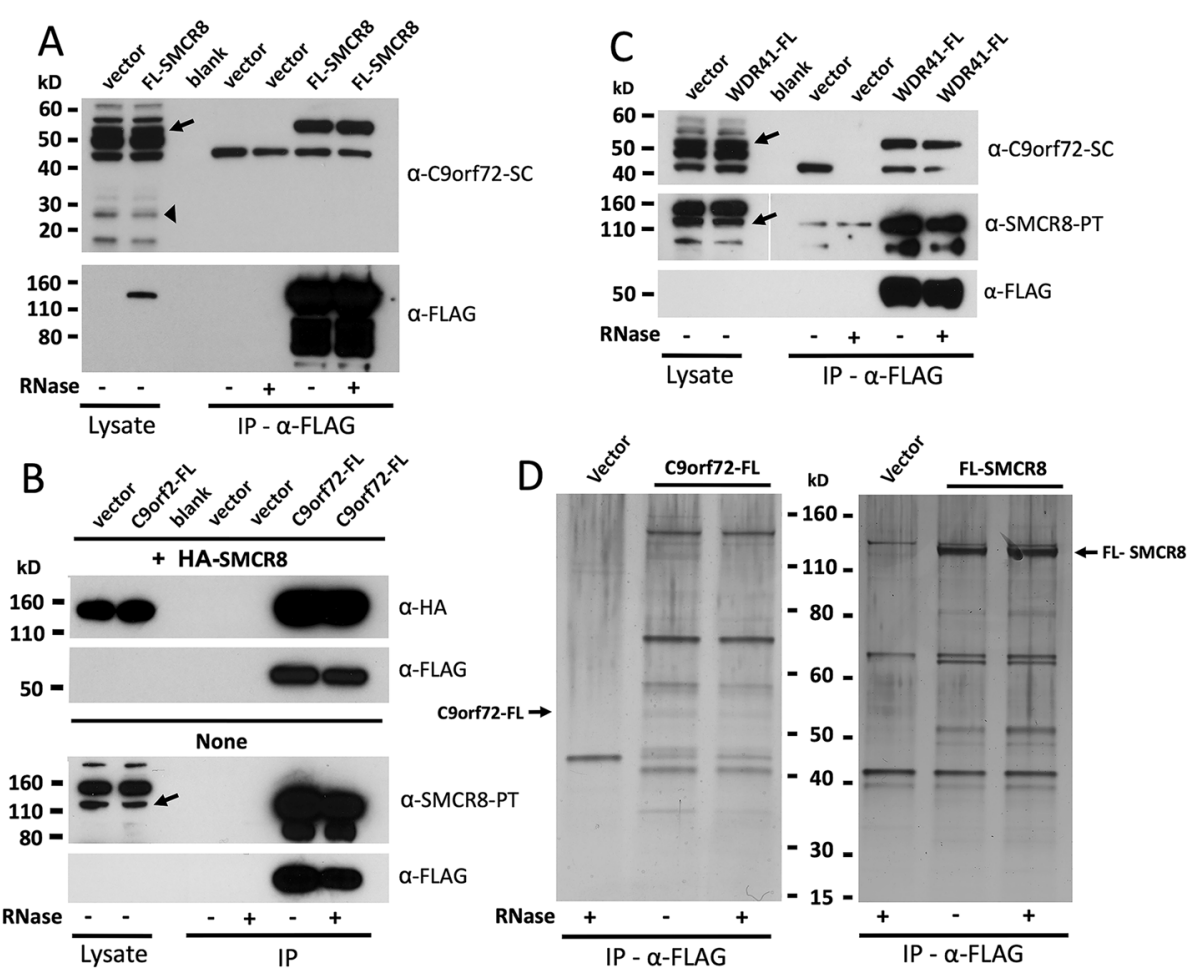

Fig. 1 Protein interaction analyses by Western blotting and co-IP of the SMCR8 complex in HEK 293T cells (see Fig. S1 for antibody analyses). a Endogenous C9-L (arrow) Co-IPs with FLAG-tagged SMCR8. The thick arrowhead marks a band consistent in size with C9-S. b FLAG-tagged C9orf72 co-IPs both endogenous and co-transfected HA-tagged SMCR8. c FLAG-tagged WDR41 protein co-IPs both endogenous C9orf72 and SMCR8 proteins (indicated by arrows). Tagged C9orf72 and WDR41 proteins of (b) and (c) are not visible in whole cell lysates at the Western blot film exposure times shown. d C9orf72-FL, FL-SMCR8, and empty vector were immunoprecipitated on a-FLAG agarose from transfected 293T whole cell lysates, resolved on a polyacrylamide gel, and silver-stained. IP reactions were in the presence or absence of $50 \mu \mathrm{g} / \mathrm{ml}$ RNases. Complex immunoprecipitate samples were analyzed by MS sequencing. Arrows indicate full-length protein bands. Protein molecular weight markers are those of Novex Sharp Pre-stained Protein Standard (Thermo Fisher Scientific)

observed for human SMCR8 [36]. The Proteintech, Bethyl (A304-694A), and Abcam (ab186504 and ab202283) $\alpha$-SMCR8 antibodies all marked a band consistent in size with full-length SMCR8 (i.e. $105 \mathrm{kD}$, arrows in Fig. S1B-E), plus additional bands of unknown specificicy, but which could in part relate to the above desribed SMCR8 protein isoforms. The Bethyl and Abcam ab202283 $\alpha$-SMCR8 antibodies have been used in other studies, and our observations are similar [34, $50,65,79]$. Interestingly, although expression of full length SMCR8 protein was detected in human brain tissues, none was seen in spinal cord tissue lysates of multiple samples (Fig. S1B-E).

WDR41 has two predicted isoforms of 51.7 and 45.5 kD (Swiss Prot. Q9HAD4-1, Q9HAD4-2). For selected cancer cell lines, both the Santa Cruz (S-12) and Proteintech (26817-1-AP) polyclonal antibodies detected doublet bands consistent in size with these isoforms (Fig. S1F, G). These bands were very faint (Proteintech) or absent (Santa Cruz) from human brain and spinal cord tissue lysates, although bands of larger and smaller sizes were visible by Western blotting of cultured cells.

\section{SMCR8 interactome contains many central nervous system (CNS) disease proteins}

Because of the possible non-specific protein interactions described above, we considered commercial antibodies unsuitable for co-IP interactome studies. Therefore, we exploited a co-IP/MS protocol that we have successfully used in previous studies [75, 76]. We transfected C9-L with C-terminal FLAG (FL)-tag, full-length SMCR8 with $\mathrm{N}$-terminal FLAG-tag, or empty vector control in HEK $293 \mathrm{~T}$ cells and performed $\alpha$-FLAG IP from whole cell extracts in the presence or absence of RNase (Fig. 1d). Complex immunoprecipitated samples were analyzed by liquid chromatography tandem MS. After excluding ribosomal proteins and likely contaminants (such as keratins), 340 and 201 proteins having three or more spectra and not detected in vector only control cell lysates were associated with FL-SMCR8 and C9-L-FL, 
Table 1 Summary of the C9orf72 and SMCR8 protein interactomes and selected functional categories

\begin{tabular}{lll}
\hline Gene Name $\quad$ Protein Name & $\frac{\text { Total \# Spectra }}{\text { RNase }}$ \\
& $\frac{-}{-}$ \\
\hline
\end{tabular}

\section{A. C9orf72-FL}

C9orf72 chromosome 9 open reading frame 72

\section{TOP 20 C9orf72-INTERACTING PROTEINS}

SMCR8

WDR41

FLG2

LRPPRC

IARS

SERPINB3

CALML5

HADHA

CDSN

EEF2

FABP5

COPB1

DSC1

U2AF2

LUC7L3

EIF2S3

HNRNPH2

KHDRBS1

PSMC2

\section{B. FL-SMCR8}

SMCR8

TOP 25 SMCR8-INTERACTING PROTEINS

DNAJA1

GTF2I

CAD

ATP1A1

EPRS

MAGED1

WDR41

DNAJC7

SLC25A3

STUB1

GCN1

DDB1

DYNC1H1

CHD4

SLC25A11

RANBP2

RUVBL2
Smith-Magenis syndrome chromosome region, candidate 8

WD repeat domain 41

filaggrin family member 2

leucine rich pentatricopeptide repeat containing

isoleucyl-tRNA synthetase

serpin family B member 3

calmodulin like 5

hydroxyacyl-CoA dehydrogenase/3-ketoacyl-CoA thiolase/enoyl-CoA hydratase, alpha subunit

corneodesmosin

eukaryotic translation elongation factor 2

fatty acid binding protein 5

coatomer protein complex subunit beta 1

desmocollin 1

U2 small nuclear RNA auxiliary factor 2

LUC7 like 3 pre-mRNA splicing factor

eukaryotic translation initiation factor 2 subunit gamma

heterogeneous nuclear ribonucleoprotein $\mathrm{H} 2$

$\mathrm{KH}$ RNA binding domain containing, signal transduction associated 1

proteasome 265 subunit, ATPase 2

$\begin{array}{ll}165 & 217 \\ 69 & 85 \\ 33 & 6 \\ 23 & 25 \\ 21 & 22 \\ 21 & 0 \\ 20 & 0 \\ 18 & 14 \\ 17 & 8 \\ 15 & 18 \\ 15 & 0 \\ 14 & 14 \\ 14 & 15 \\ 14 & 6 \\ 13 & 4 \\ 12 & 10 \\ 12 & 9 \\ 12 & 6 \\ 12 & 25\end{array}$

1135

DnaJ heat shock protein family member A1

general transcription factor IIi

70

55

62

carbamoyl-phosphate synthetase 2, aspartate transcarbamylase, and dihydroorotase

ATPase $\mathrm{Na}+/ \mathrm{K}+$ transporting subunit alpha 1

glutamyl-prolyl-tRNA synthetase

MAGE family member D1

WD repeat domain 41

DnaJ heat shock protein family member C7

solute carrier family 25 member 3

STIP1 homology and U-box containing protein 1

GCN1, elF2 alpha kinase activator homolog

damage specific DNA binding protein 1

dynein cytoplasmic 1 heavy chain 1

chromodomain helicase DNA binding protein 4

solute carrier family 25 member 11

RAN binding protein 2

RuvB like AAA ATPase 2

14

25

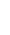 \\ 82}


Table 1 Summary of the C9orf72 and SMCR8 protein interactomes and selected functional categories (Continued)

\begin{tabular}{|c|c|c|c|}
\hline \multirow[t]{3}{*}{ Gene Name } & \multirow[t]{3}{*}{ Protein Name } & \multicolumn{2}{|c|}{ Total \# Spectra } \\
\hline & & \multicolumn{2}{|c|}{ RNase } \\
\hline & & - & + \\
\hline ATP2A2 & ATPase sarcoplasmic/endoplasmic reticulum Ca2+ transporting 2 & 19 & 22 \\
\hline DNAJA3 & DnaJ heat shock protein family member $\mathrm{A} 3$ & 19 & 22 \\
\hline IARS & isoleucyl-tRNA synthetase & 18 & 22 \\
\hline STIP1 & stress induced phosphoprotein 1 & 37 & 22 \\
\hline COPA & coatomer protein complex subunit alpha & 23 & 20 \\
\hline HUWE1 & HECT, UBA and WWE domain containing 1, E3 ubiquitin protein ligase & 29 & 20 \\
\hline AlFM1 & apoptosis inducing factor, mitochondria associated 1 & 18 & 18 \\
\hline C9orf72 & chromosome 9 open reading frame 72 & 15 & 18 \\
\hline \multicolumn{4}{|c|}{ AMINOACYL-tRNA SYNTHESIS } \\
\hline EPRS & glutamyl-prolyl-tRNA synthetase & 40 & 13 \\
\hline IARS & isoleucyl-tRNA synthetase & 18 & 22 \\
\hline DARS & aspartyl-tRNA synthetase & 11 & 8 \\
\hline MARS & methionyl-tRNA synthetase & 10 & 6 \\
\hline RARS & arginyl-tRNA synthetase & 9 & 8 \\
\hline LARS & leucyl-tRNA synthetase & 9 & 14 \\
\hline QARS & glutaminyl-tRNA synthetase & 8 & 7 \\
\hline TARS2 & threonyl-tRNA synthetase 2, mitochondrial (putative) & 6 & 4 \\
\hline TARS & threonyl-tRNA synthetase & 4 & 0 \\
\hline FARSA & phenylalanyl-tRNA synthetase alpha subunit & 0 & 6 \\
\hline \multicolumn{4}{|c|}{ CELL CYCLE } \\
\hline SMC3 & structural maintenance of chromosomes 3 & 12 & 8 \\
\hline CDK4 & cyclin dependent kinase 4 & 5 & 0 \\
\hline PCNA & proliferating cell nuclear antigen & 5 & 4 \\
\hline MCM7 & minichromosome maintenance complex component 7 & 4 & 6 \\
\hline YWHAZ & tyrosine 3-monooxygenase/tryptophan 5-monooxygenase activation protein zeta & 4 & 9 \\
\hline RAD21 & RAD21 cohesin complex component & 3 & 0 \\
\hline CDC7 & cell division cycle 7 & 3 & 0 \\
\hline PLK1 & polo like kinase 1 & 3 & 9 \\
\hline PPP6C & protein phosphatase 6 catalytic subunit & 3 & 0 \\
\hline CDKN2A & cyclin dependent kinase inhibitor 2A & 0 & 3 \\
\hline MCM2 & minichromosome maintenance complex component 2 & 0 & 4 \\
\hline MCM5 & minichromosome maintenance complex component 5 & 0 & 4 \\
\hline SMC1A & structural maintenance of chromosomes $1 \mathrm{~A}$ & 0 & 4 \\
\hline \multicolumn{4}{|c|}{ HEAT SHOCK PROTEINS / CHAPERONES } \\
\hline DNAJA1 & DnaJ heat shock protein family member A1 & 70 & 76 \\
\hline DNAJC7 & DnaJ heat shock protein family member C7 & 30 & 32 \\
\hline DNAJA3 & DnaJ heat shock protein family member $\mathrm{A} 3$ & 19 & 22 \\
\hline DNAJA3 & DnaJ heat shock protein family member $\mathrm{A} 3$ & 19 & 22 \\
\hline $\mathrm{HSPH} 1$ & heat shock protein family $\mathrm{H}$ member 1 & 19 & 8 \\
\hline BAG5 & BCL2 associated athanogene 5 & 18 & 11 \\
\hline DNAJA2 & DnaJ heat shock protein family member $\mathrm{A} 2$ & 15 & 16 \\
\hline BAG2 & BCL2 associated athanogene 2 & 11 & 16 \\
\hline
\end{tabular}


Table 1 Summary of the C9orf72 and SMCR8 protein interactomes and selected functional categories (Continued)

\begin{tabular}{|c|c|c|c|}
\hline \multirow[t]{3}{*}{ Gene Name } & \multirow[t]{3}{*}{ Protein Name } & \multicolumn{2}{|c|}{ Total \# Spectra } \\
\hline & & \multicolumn{2}{|c|}{ RNase } \\
\hline & & - & + \\
\hline BAG6 & BCL2 associated athanogene 6 & 11 & 8 \\
\hline SERPINH1 & serpin family $\mathrm{H}$ member 1 & 11 & 0 \\
\hline HSPB1 & heat shock protein family B member 1 & 10 & 9 \\
\hline MDN1 & midasin AAA ATPase 1 & 10 & 14 \\
\hline FKBP8 & FK506 binding protein 8 & 8 & 0 \\
\hline DNAJB6 & DnaJ heat shock protein family member B6 & 6 & 4 \\
\hline PFDN2 & prefoldin subunit 2 & 5 & 4 \\
\hline DNAJB1 & DnaJ heat shock protein family member B1 & 4 & 0 \\
\hline HSPA4 & heat shock protein family A member 4 & 4 & 3 \\
\hline HSPA4 & heat shock protein family A member 4 & 4 & 3 \\
\hline RBBP7 & RB binding protein 7 , chromatin remodeling factor & 3 & 4 \\
\hline SERPINB3 & serpin family B member 3 & 3 & 0 \\
\hline BAG3 & BCL2 associated athanogene 3 & 2 & 3 \\
\hline UNC45A & unc-45 myosin chaperone $\mathrm{A}$ & 2 & 5 \\
\hline DNAJB11 & DnaJ heat shock protein family member B11 & 0 & 6 \\
\hline DNAJC10 & DnaJ heat shock protein family member $\mathrm{C} 10$ & 0 & 5 \\
\hline PARK7 & Parkinsonism associated deglycase & 0 & 4 \\
\hline \multicolumn{4}{|c|}{ MISMATCH REPAIR } \\
\hline RFC3 & replication factor $C$ subunit 3 & 5 & 0 \\
\hline SSBP1 & single stranded DNA binding protein 1 & 5 & 0 \\
\hline MSH6 & mutS homolog 6(MSH6) & 5 & 5 \\
\hline POLD3 & DNA polymerase delta 3, accessory subunit & 2 & 3 \\
\hline PCNA & proliferating cell nuclear antigen & 0 & 4 \\
\hline POLD1 & DNA polymerase delta 1 , catalytic subunit & 0 & 3 \\
\hline
\end{tabular}

\section{PHAGOSOME/AUTOPHAGY}

DYNC1H1

SEC16A

TUBB4A

IRS4

TUBB3

TUBB6

PPP2R1A

CDK5

SEC61B

HLA-A

ANKRD28

RAB1B

SLC3A2

SEC24C

TFRC

AP3M1

PPP6C dynein cytoplasmic 1 heavy chain 1

SEC16 homolog A, endoplasmic reticulum export factor

tubulin beta 4A class IVa

insulin receptor substrate 4

tubulin beta 3 class III

tubulin beta 6 class $V$

protein phosphatase 2 scaffold subunit Aalpha

cyclin dependent kinase 5

Sec61 translocon beta subunit

major histocompatibility complex, class I, A

aminoadipate-semialdehyde dehydrogenase-phosphopantetheinyl transferase

RAB1B, member RAS oncogene family

solute carrier family 3 member 2

SEC24 homolog C, COPII coat complex component

transferrin receptor

adaptor related proteincomplex $3 \mathrm{Mu} 1$ subunit

protein phosphatase 6 catalytic subunit
38 
Table 1 Summary of the C9orf72 and SMCR8 protein interactomes and selected functional categories (Continued)

\begin{tabular}{ll}
\hline Gene Name & Protein Name \\
\hline BAG3 & BCL2 associated athanogene 3 \\
WDR5 & WD repeat domain 5 \\
YME1L1 & YME1 like 1 ATPase \\
KEAP1 & kelch like ECH associated protein 1 \\
DYNC112 & dynein cytoplasmic 1 intermediate chain 2 \\
CDKN2A & Cyclin dependent kinase inhibitor 2A \\
HLA-B & major histocompatibility complex, class I, B
\end{tabular}

\begin{tabular}{ll}
\multicolumn{2}{l}{ Total \# Spectra } \\
\hline RNase & \\
\hline- & + \\
\hline 2 & 3 \\
0 & 5 \\
0 & 4 \\
0 & 4 \\
0 & 4 \\
0 & 3 \\
0 & 3
\end{tabular}

PROTEIN PROCESSING IN THE ENDOPLASMIC RETICULUM

DNAJA1

STUB1

$\mathrm{HSPH} 1$

DNAJA2

BAG2

SEC61B

RPN1

TXNDC5

DNAJB1

SEC24C

DDOST

SSR4

DNAJB11

DNAJC10

RRBP1

\section{PROTEOSOME}

PSMC2

PSMD2

PSMA7

PSMD11

PSMC3

PSMD3

PSMA5

PSMC5

PSME3

PSMA6

PSMD6

PSMA3

PSMD1

PSMA1

PSMB1

PSMB5

PSMC1

PSMB2

PSMB6
DnaJ heat shock protein family (Hsp40) member A1

STIP1 homology and U-box containing protein 1

heat shock protein family $\mathrm{H}$ (Hsp 110) member 1

DnaJ heat shock protein family (Hsp40) member A2

BCL2 associated athanogene 2

Sec61 translocon beta subunit

ribophorin I

thioredoxin domain containing 5

DnaJ heat shock protein family (Hsp40) member B1

SEC24 homolog C, COPII coat complex component

dolichyl-diphosphooligosaccharide--protein glycosyltransferase 48 kD subunit

signal sequence receptor subunit 4

DnaJ heat shock protein family (Hsp40) member B11

DnaJ heat shock protein family (Hsp40) member C10

ribosome binding protein 1

proteasome 265 subunit, ATPase 2

proteasome 265 subunit, non-ATPase 2

proteasome subunit alpha 7

proteasome 265 subunit, non-ATPase 11

proteasome 265 subunit, ATPase 3

proteasome $26 \mathrm{~S}$ subunit, non-ATPase 3

proteasome subunit alpha 5

proteasome 26S Subunit, ATPase 5

proteasome activator subunit 3

proteasome subunit alpha 6

proteasome 265 subunit, non-ATPase 6

proteasome subunit alpha 3

proteasome 265 subunit, non-ATPase 1

proteasome subunit alpha 1

proteasome subunit beta 1

proteasome subunit beta 5

proteasome 265 subunit, ATPase 1

proteasome subunit beta 2

proteasome subunit beta 6

$\begin{array}{ll}70 & 76 \\ 30 & 31 \\ 19 & 8 \\ 15 & 16 \\ 11 & 16 \\ 6 & 0 \\ 5 & 3 \\ 5 & 2 \\ 4 & 0 \\ 4 & 3 \\ 3 & 4 \\ 2 & 3 \\ 0 & 6 \\ 0 & 5 \\ 0 & 3\end{array}$

76

8

16

3

2

0

3

4

3

6

5

$22 \quad 18$

$18 \quad 10$

$17 \quad 16$

149

129

$12 \quad 8$

$11 \quad 6$

$10 \quad 10$

$10 \quad 11$

90

$9 \quad 7$

83

$8 \quad 8$

$\begin{array}{ll}7 & 7\end{array}$

$7 \quad 7$

$6 \quad 6$

$6 \quad 6$

55

$5 \quad 5$ 
Table 1 Summary of the C9orf72 and SMCR8 protein interactomes and selected functional categories (Continued)

\begin{tabular}{|c|c|c|c|}
\hline \multirow[t]{3}{*}{ Gene Name } & \multirow[t]{3}{*}{ Protein Name } & \multicolumn{2}{|c|}{ Total \# Spectra } \\
\hline & & \multicolumn{2}{|c|}{ RNase } \\
\hline & & - & + \\
\hline PSMC4 & proteasome 265 subunit, ATPase 4 & 5 & 0 \\
\hline PSMC6 & proteasome $26 S$ subunit, ATPase 6 & 5 & 6 \\
\hline PSMD12 & proteasome $26 \mathrm{~S}$ subunit, non-ATPase 12 & 4 & 2 \\
\hline PSMD7 & proteasome $26 \mathrm{~S}$ subunit, non-ATPase 7 & 3 & 0 \\
\hline PSMD13 & proteasome $26 \mathrm{~S}$ subunit, non-ATPase 13 & 0 & 3 \\
\hline PSMD14 & proteasome $26 \mathrm{~S}$ subunit, non-ATPase 14 & 0 & 3 \\
\hline PSMD8 & proteasome $26 \mathrm{~S}$ subunit, non-ATPase 8 & 0 & 6 \\
\hline \multicolumn{4}{|c|}{ RNA TRANSPORT } \\
\hline RANBP2 & RAN binding protein 2 & 29 & 25 \\
\hline XPO1 & exportin 1 & 13 & 13 \\
\hline NUP93 & nucleoporin 93 & 11 & 10 \\
\hline NUP133 & nucleoporin 133 & 10 & 15 \\
\hline THOC2 & THO complex 2 & 9 & 6 \\
\hline RANGAP1 & Ran GTPase activating protein 1 & 8 & 11 \\
\hline KPNB1 & karyopherin subunit beta 1 & 7 & 5 \\
\hline KPNA2 & karyopherin subunit alpha 2 & 6 & 4 \\
\hline ElF4A1 & eukaryotic translation initiation factor 4A1 & 5 & 4 \\
\hline ElF4G1 & eukaryotic translation initiation factor 4 gamma 1 & 5 & 4 \\
\hline NUP155 & nucleoporin 155 & 4 & 6 \\
\hline NUP210 & nucleoporin 210 & 4 & 0 \\
\hline EIF3F & eukaryotic translation initiation factor 3 subunit $F$ & 3 & 3 \\
\hline FXR2 & FMR1 autosomal homolog 2 & 3 & 0 \\
\hline NUP160 & nucleoporin 160 & 3 & 4 \\
\hline NUP205 & nucleoporin 205 & 3 & 4 \\
\hline EIF3E & eukaryotic translation initiation factor 3 subunit $E$ & 2 & 4 \\
\hline FMR1 & fragile $\mathrm{X}$ mental retardation 1 & 2 & 0 \\
\hline NUP98 & nucleoporin 98 & 2 & 5 \\
\hline NUP153 & nucleoporin 153 & 0 & 5 \\
\hline THOC3 & THO complex 3 & 0 & 4 \\
\hline XРОТ & exportin for tRNA & 0 & 4 \\
\hline \multicolumn{4}{|c|}{ UBIQUITINATION / SUMOYLATION } \\
\hline STUB1 & STIP1 homology and U-box containing protein 1 & 30 & 31 \\
\hline HUWE1 & HECT, UBA and WWE domain containing 1, E3 ubiquitin ligase & 29 & 20 \\
\hline RANBP2 & RAN binding protein 2, E3 SUMO ligase & 29 & 25 \\
\hline RNF40 & ring finger protein 40, E3 ubiquitin ligase & 4 & 10 \\
\hline USP7 & ubiquitin specific peptidase 7 & 4 & 9 \\
\hline USP9X & ubiquitin specific peptidase $9, X$-linked & 4 & 5 \\
\hline RNF2 & ring finger protein 2, , E3 ubiquitin protein ligase & 3 & 0 \\
\hline UBR5 & ubiquitin protein ligase E3 component n-recognin 5 & 3 & 12 \\
\hline USP15 & ubiquitin specific peptidase 15 & 3 & 0 \\
\hline SUMO3 & small ubiquitin-like modifier 3 & 0 & 3 \\
\hline MKRN2 & makorin ring finger protein 2, E3 ubiquitin ligase & 0 & 3 \\
\hline
\end{tabular}


respectively (Tables 1, S1, S2). Furthermore, 71 proteins were found in both proteomes, although it should be noted that C9-L-FL was expressed at significantly lower levels than FL-SMCR8 (Fig. 1d), as previously reported [29]. Tables S1 and S2 also note interacting partners of C9orf72 or SMCR8 proteins reported in previous studies $[19,28,29,31,32,34,51,87,88]$. During the course of our investigations, another MS experiment was published that listed 1532 proteins that co-IPed with HA-tagged SMCR8 from 293T cells [34], and a total of 272 of these $(80 \%)$ were also present in our dataset (Table S2).

To further confirm the effectiveness of our MS analyses, we next analyzed some of the interactors identified. To do that, a subset of cDNAs identified from the SMCR8 proteome were cloned with an N-terminal V5TEV (tobacco etch virus)-epitope tag or were obtained as gifts. Notably, following cotransfection in 293T cells, $73 \%(22 / 30)$ of proteins tested directly co-IPed with FLSMCR8 on $\alpha$-FLAG agarose, further confirming the efficiency of our protocol (Fig. 2). In almost all cases, interactions were resistant to RNase digestion. Some proteins bound non-specifically to the agarose (BAG5, PPP2R1A, RUVBL2) or failed to bind FL-SMCR8 (G3BP1, GTF2I, RAB1A, RANGAP1, STIP1). It is possible some of these latter proteins are only able to bind SMCR8 when in complex with over-expressed C9orf72 and/or WDR41.

Several studies have proposed a role for C9orf72 in the regulation of autophagy by Rab GTPases, although with disagreement concerning which of the many Rab family members binds the C9orf72/SMCR8/WDR41 complex. Farg et al. [43] first reported C9orf72 to interact with RAB1, RAB5, RAB7 and RAB11. Webster et al. [22] confirmed that C9orf72 associates with GTP-bound RAB1A and the ULK1 complex, and it has been demonstrated that C9orf72 in complex with SMCR8 and WDR41 is a GEF for RAB8A, RAB11A, and RAB39B, and that its loss perturbs autophagy in neurons [27, 29, 31, 89]. We detected only RAB1B in our SMCR8 and C9orf72 interactomes (Tables S1, S2), but failed to confirm binding of V5-tagged RAB1A, a paralog highly similar in sequence to RAB1A, with SMCR8 in direct co-IP experiments. However, we also tested and confirmed weak binding of V5-RAB7A with overexpressed SMCR8 (Fig. 2) and C9orf72 (not shown), but only in the presence of RNase.

Significantly, when we queried the OMIM (Online Mendelian Inheritence in Man) database (https://omim. org/), we found that 65 (19\%) of our putative SMCR8interacting proteins are associated with neurodegenerative and neurological genetic disorders (Table 2). These include 8 proteins linked with ALS and/or FTD, 14 with other neurodegenerative diseases (including 4 associated with spinocerebellar ataxias), 7 with Charcot-Marie Tooth disease, 5 with hypomyelinating leukodystrophy, and 13 with mental retardation. Thus, SMCR8 may recruit some of these proteins to its complex with C9orf72 and WDR41, predicting roles for the complex in central nervous system (CNS) disorders.

\section{SMCR8 associates with proteins of the ubiquitination and protein decay pathways}

Our MS analyses also showed that both SMCR8 and C9orf72 proteins bind the proteasome. Our lists of SMCR8- and C9orf72-interactors were examined for KEGG pathways using the DAVID Functional Annotation Tool (NIAID, NIH, [83]). In the case of SMCR8-associated proteins, proteasome $(7.6 \%$ of total proteins), RNA transport (6.2\%), and protein processing in the endoplasmic reticulum (4.4\%) pathways predominated (Fig. S2A). For C9orf72, proteins associated with the proteasome and RNA transport pathways accounted for 4.5 and $8 \%$ of the total, respectively, while spliceosome pathway proteins (9\%) were most abundant (Fig. S2B). Twenty-six proteasome subunits co-IPed with FL-SMCR8, and 9 subunits were detected within the C9orf72-FL interactome. Furthermore, based on KEGG and Autophagy Database (http://www.tanpaku.org/autophagy/) analyses, 24 proteins involved with phagosome and autophagy pathways were detected in the SMCR8 interactome, including proteins linked with neurological diseases (CDK5, DYNC1H1, HUWE1, PPP2R1A, RANBP2, STUB1, TUBB3, TUBB4A, and USP9X; Tables 1, 2, S2).

Our SMCR8 interactome also contained 9 ubiquitination pathway factors, including ubiquitin ligases and peptidases (Table 1). Therefore, we examined MSsequenced peptides deriving from immunoprecipitated FL-SMCR8 for ubiquitin modification (72\% coverage of the total protein). A total of 9 high confidence modified lysine residues were predicted by at least 5 peptides in two independent experiments, suggesting that SMCR8 is highly ubiquitinated. Eight of these lysines were also identified by at least one of three ubiquitination prediction algorithms, including UbPred [86], BDM-PUB (http://bdmpub.biocuckoo.org), and UbiSite (http://csb.cse.yzu.edu.tw/UbiSite/) (Table S3). We then considered the phylogenic conservation of these lysines by aligning SMCR8 protein sequences from 8 vertebrate (human, chimpanzee, dog, mouse, rat, chicken, zebrafish, and frog) and two mollusc (freshwater snail and sea slug) species (Fig. S3). Eight of the 9 lysines detected by MS as modified were conserved among at least 8 species, including 2 residues (K232, K479) found in both molluscs, suggesting that these post-translational modifications (PTMs) might be functionally relevant.

Immunoprecipitating FLAG-tagged SMCR8 and probing with $\alpha$-ubiquitin on Western blots reveals highmolecular weight (HMW) proteins consistent with 

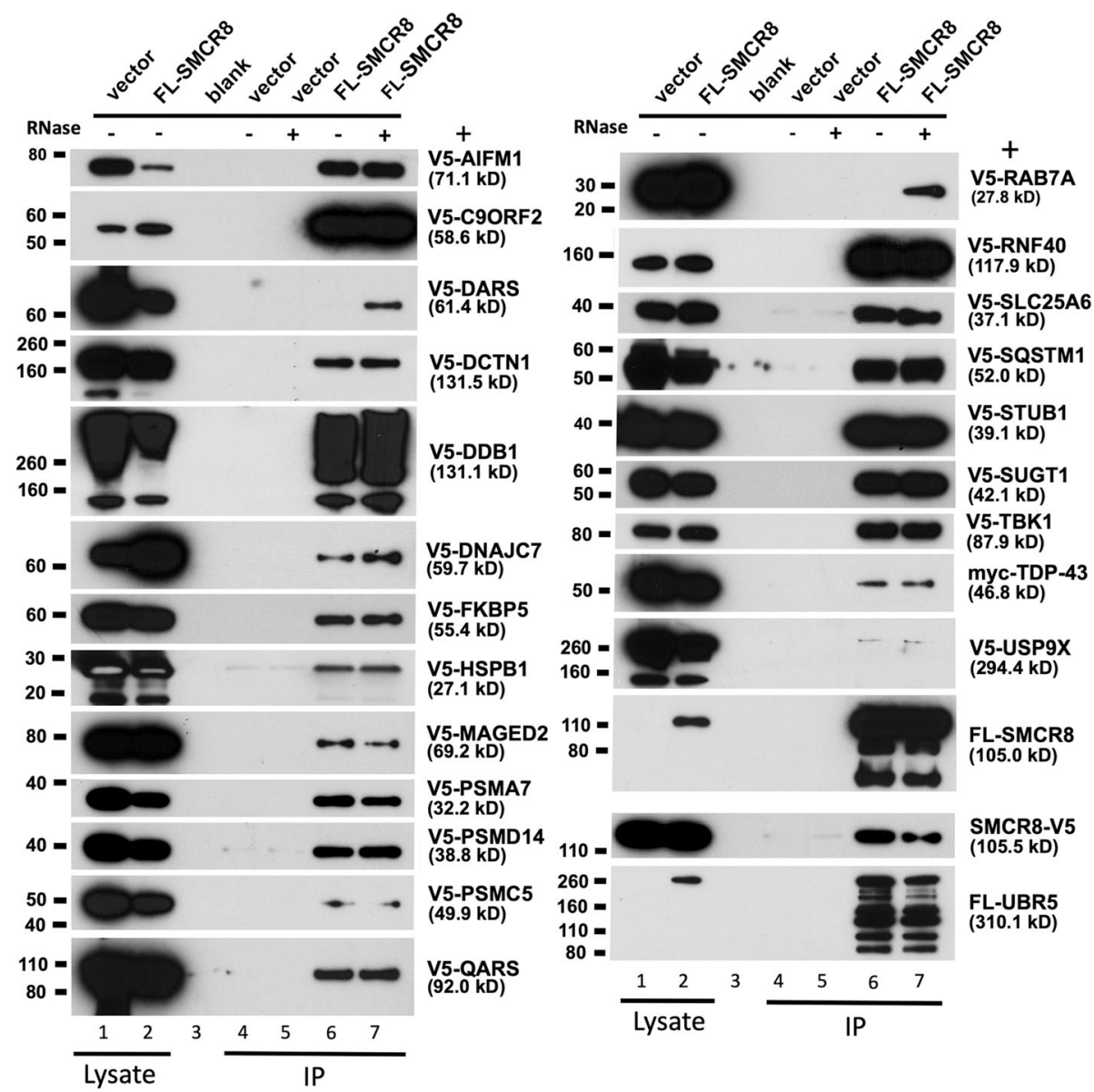

Fig. 2 Confirmation of proteins in the SMCR8 complex. Selected proteins detected in the SMCR8 interactome by MS sequencing were tagged and coexpressed with SMCR8 in HEK 293T cells. Most were found to specifically co-IP on a-FLAG agarose with FL-SMCR8 but not empty vector. Approximately $1 \%$ of the input lysate (lanes 1,2) and 30\% of the immunoprecipitate (lanes $4-7$ ) were loaded on gels. IP reactions were in the presence or absence of $50 \mu \mathrm{g} / \mathrm{ml}$ RNases. Also included is a panel representative of tagged FL-SMCR8 protein present in the input and IP fractions (detected by a-FLAG antibody) and showing that RNase treatment did not affect SMCR8 immunoprecipitation (lower right). Test proteins were detected by a-V5 antibody, except FL-UBR5, which was detected by a-FLAG antibody (bottom right). The molecular weight of each test protein, including its epitope tag, is shown in brackets. Protein molecular weight markers are those of Novex Sharp Pre-stained Protein Standard (Thermo Fisher)

polyubiquitinated SMCR8 and/or other large ubiquitinated proteins bound in the SMCR8 complex (Fig. 3a). In whole cell lysates, SMCR8-V5, in the presence of the proteasome inhibitor MG-132 and/or coexpressed ubiquitin, showed HMW products consistent with multiple PTMs (Fig. 3b). Furthermore, FLAG-tagged ubiquitin coimmunoprecipitates on $\alpha$-FLAG agarose, and so by implication is conjugated to cotransfected HA- or V5tagged SMCR8 (Fig. 3c). Although treatment with MG132 caused accumulation of HMW SCMR8 protein species, suggesting their regulation by the ubiquitinproteasome system (UPS), full-length SMCR8 signal was little decreased in the presence of coexpressed ubiquitin (Fig. 3b,c).
Using confocal IF microsopy, we observed that overexpression of red fluorescent protein (RFP)-tagged ubiquitin induces formation of a large aggregate consistent with the aggresome and marked by colocalization with coexpressed and therefore likely UBB-bound FL-SMCR8 (Fig. 4a). Aggresomes appear mainly within an indentation of the nucleus at the microtubule-organizing center and form when the protein-degradation machinery of the cell is overwhelmed [90]. Misfolded and ubiquitinated proteins, including perhaps SMCR8, are transported to the aggresome along the microtubule network by means of the dynein motor complex (which includes cytoplasmic dyneins DYNC1H1 and DYNC1I2, both detected in FL-SMCR8 iimmunoprecipitates, Table S2). An 
Table 2 Proteins of the SMCR8 interactome associated with CNS disease according to the Online Mendelian Inheritance in Man (OMIM) database

\begin{tabular}{|c|c|c|c|}
\hline GENE NAME & DISEASE NAME & SYMBOL & OMIM \# \\
\hline \multicolumn{4}{|l|}{ ALS/FTD } \\
\hline ATXN2 & Amyotrophic lateral sclerosis 13 & ALS13 & 183,090 \\
\hline C9orf72 & Frontotemporal dementia and/or amyotrophic lateral sclerosis 1 & FTDALS1 & 105,550 \\
\hline DCTN1 & Amyotrophic lateral sclerosis & ALS & 105,400 \\
\hline FIG4 & Amyotrophic lateral sclerosis 11 & ALS11 & 612,577 \\
\hline FUS & Amyotrophic lateral sclerosis 6 , with or without frontotemporal dementia & ALS6 & 608,030 \\
\hline MATR3 & Amyotrophic lateral sclerosis 21 & ALS21 & 606,070 \\
\hline SQSTM1 & Frontotemporal dementia and/or amyotrophic lateral sclerosis 3 & FTDALS3 & 616,437 \\
\hline TARDBP & Amyotrophic lateral sclerosis 10 & ALS10 & 612,069 \\
\hline \multicolumn{4}{|c|}{ OTHER NEURODEGENERATION DISEASES } \\
\hline AlFM1 & Combined oxidative phosphorylation deficiency 6 & COXPD6 & 300,816 \\
\hline ATXN2 & Spinocerebellar ataxia 2 & SCA2 & 183,090 \\
\hline CTSD & Ceroid lipofuscinosis, neuronal, 10 & CLN10 & 610,127 \\
\hline DYNC1H1 & Spinal muscular atrophy, lower extremity-predominant 1, autosomal dominant & SMALED1 & 158,600 \\
\hline EIF4G1 & Parkinson disease 18 & PARK18 & 614,251 \\
\hline NOP56 & Spinocerebellar ataxia 36 & SCA36 & 614,153 \\
\hline PARK7 & Parkinson disease 7 & PARK7 & 606,324 \\
\hline PCNA & Ataxia-telangiectasia-like disorder 2 & ATLD2 & 615,919 \\
\hline PUM1 & Spinocerebellar ataxia 47 & SCA47 & 617,931 \\
\hline QARS & Microcephaly, progressive, with seizures and cerebral and cerebellar atrophy & MSCCA & 615,760 \\
\hline RARS & Leukodystrophy, hypomyelinating, 9 & HLD9 & 616,140 \\
\hline SPG20 & Spastic paraplegia 20, autosomal recessive & SPG20 & 275,900 \\
\hline STUB1 & Spinocerebellar ataxia, autosomal recessive, 16 & SCAR16 & 615,768 \\
\hline WNK1 & Neuropathy, hereditary sensory and autonomic, 2A & HSAN2A & 201,300 \\
\hline \multicolumn{4}{|c|}{ OTHER NEUROLOGICAL CONDITIONS } \\
\hline ADAR & Aicardi-Goutieres syndrome 6 & AGS6 & 615,010 \\
\hline ADNP & Helsmoortel-van der Aa syndrome & HVDAS & 615,873 \\
\hline AIFM1 & Cowchock syndrome & COWCK & 310,490 \\
\hline AIMP1 & Leukodystrophy, hypomyelinating, 3 & HLD3 & 260,600 \\
\hline AIMP2 & Leukodystrophy, hypomyelinating, 17 & HLD17 & 618,006 \\
\hline $\mathrm{ALDH} 18 \mathrm{~A} 1$ & Spastic paraplegia 9A, autosomal dominant & SPG9A & 601,162 \\
\hline $\mathrm{ALDH} 18 \mathrm{~A} 1$ & Spastic paraplegia 9B, autosomal recessive & SPG9B & 616,586 \\
\hline $\mathrm{ALDH} 3 \mathrm{~A} 2$ & Sjogren-Larsson syndrome & SLS & 270,200 \\
\hline ARHGEF2 & Neurodevelopmental disorder with midbrain and hindbrain malformations & NEDMHM & 617,523 \\
\hline ATAD3A & Harel-Yoon syndrome & ATAD3A & 612,316 \\
\hline ATP1A1 & Charcot-Marie-Tooth disease, axonal, type 2DD & CMT2DD & 618,036 \\
\hline CAD & Epileptic encephalopathy, early infantile, 50 & $A R$ & 616,457 \\
\hline CDK5 & Lissencephaly 7, with cerebellar hypoplasia & LIS7 & 616,342 \\
\hline CLTC & Mental retardation, autosomal dominant 56 & MRD56 & 617,854 \\
\hline COPB2 & Microcephaly 19, primary, autosomal recessive & MCPH19 & 617,800 \\
\hline DARS & Hypomyelination with brainstem and spinal cord involvement and leg spasticity & HBSL & 615,281 \\
\hline DCTN1 & Neuronopathy, distal hereditary motor, 7B & HMN7B & 607,641 \\
\hline DCTN1 & Perry syndrome & PERRYS & 168,605 \\
\hline
\end{tabular}


Table 2 Proteins of the SMCR8 interactome associated with CNS disease according to the Online Mendelian Inheritance in Man (OMIM) database (Continued)

\begin{tabular}{|c|c|c|c|}
\hline GENE NAME & DISEASE NAME & SYMBOL & OMIM \# \\
\hline DOCK7 & Epileptic encephalopathy, early infantile, 23 & $A R$ & 615,859 \\
\hline DYNC1H1 & Charcot-Marie-Tooth disease 20 & СMT2O & 614,228 \\
\hline DYNC1H1 & Mental retardation, autosomal dominant 13 & MRD13 & 614,563 \\
\hline EPRS & Leukodystrophy, hypomyelinating, 15 & HLD15 & 617,951 \\
\hline FIG4 & Polymicrogyria, bilateral temporooccipital & BTOP & 612,691 \\
\hline FIG4 & Charcot-Marie-Tooth disease 4J & CMT4J & 611,228 \\
\hline FKBP5 & \{Major depressive disorder and accelerated response to antidepressant drugs\} & MDD & 608,516 \\
\hline FMR1 & Fragile $X$ syndrome & FRAX & 300,624 \\
\hline FMR1 & Fragile $X$ tremor/ataxia syndrome & FXTAS & 300,623 \\
\hline GATAD2B & Mental retardation, autosomal dominant 18 & MRD18 & 615,074 \\
\hline HCFC1 & Mental retardation, $\mathrm{X}$-linked 3 & MRX3 & 309,541 \\
\hline HNRNPH2 & Mental retardation, X-linked, syndromic, Bain type & MRXSB & 300,986 \\
\hline HPRT1 & Lesch-Nyhan syndrome & LNS & 300,322 \\
\hline HSD17B4 & Perrault syndrome 1 & PRLTS1 & 233,400 \\
\hline HSPB1 & Neuropathy, distal hereditary motor, type IIB & HMN2B & 608,634 \\
\hline HSPB1 & Charcot-Marie-Tooth disease 2F & $\mathrm{CMT} 2 \mathrm{~F}$ & 606,595 \\
\hline HUWE1 & Mental retardation, X-linked 17 & MRX17 & 300,705 \\
\hline HUWE1 & Mental retardation, X-linked, syndromic, Turner type & MRXST & 300,706 \\
\hline LAS1L & Wilson-Turner syndrome & WTS & 309,585 \\
\hline LMNA & Charcot-Marie-Tooth disease 2B1 & CMT2B1 & 605,588 \\
\hline LRPPRC & Leigh syndrome French-Canadian type & LSFC & 220,111 \\
\hline MARS & Charcot-Marie-Tooth disease $2 U$ & CMT2U & 616,280 \\
\hline MTHFD1 & Neural tube defects, folate-sensitive & NTDFS & 601,634 \\
\hline NSUN2 & Mental retardation, autosomal recessive 5 & MRT5 & 611,091 \\
\hline PDK3 & Charcot-Marie-Tooth disease, X-linked dominant, 6 & СMTX6 & 300,905 \\
\hline PPP2R1A & Mental retardation, autosomal dominant 36 & MRD36 & 616,362 \\
\hline PSMD12 & Stankiewicz-Isidor syndrome & STISS & 617,516 \\
\hline RANBP2 & Encephalopathy, acute, infection-induced, 3 & IIAE3 & 608,033 \\
\hline SAMHD1 & Aicardi-Goutieres syndrome 5 & AGS5 & 612,952 \\
\hline SLC25A1 & Combined D-2- and L-2-hydroxyglutaric aciduria & D2L2AD & 615,182 \\
\hline SLC25A22 & Epileptic encephalopathy, early infantile, 3 & EIEE3 & 609,304 \\
\hline SPTLC1 & Neuropathy, hereditary sensory and autonomic, type IA & HSN1A & 162,400 \\
\hline TECR & Mental retardation, autosomal recessive 14 & MRT14 & 614,020 \\
\hline THOC2 & Mental retardation, X-linked 12 & $\operatorname{MRX} 12$ & 300,957 \\
\hline TUBB3 & Cortical dysplasia, complex, with other brain malformations 1 & CDCBM1 & 614,039 \\
\hline TUBB4A & Leukodystrophy, hypomyelinating, 6 & HLD & 612,438 \\
\hline USP9X & Mental retardation, X-linked 99 & MRX99 & 300,919 \\
\hline USP9X & Mental retardation, X-linked 99, syndromic, female-restricted & MRXS99F & 300,968 \\
\hline
\end{tabular}

alternative ubiquitin-independent pathway involves interaction of STUB1 and BAG3, which transfer misfolded proteins to heat shock protein 70 (all proteins that co-IPed with FL-SMCR8, Table S2) and the dynein motor complex to promote formation of aggresomes $[91,92]$.

Thus, SMCR8 protein is bound by ubiquitin and may recruit UPS complexes to the vicinity of its other 


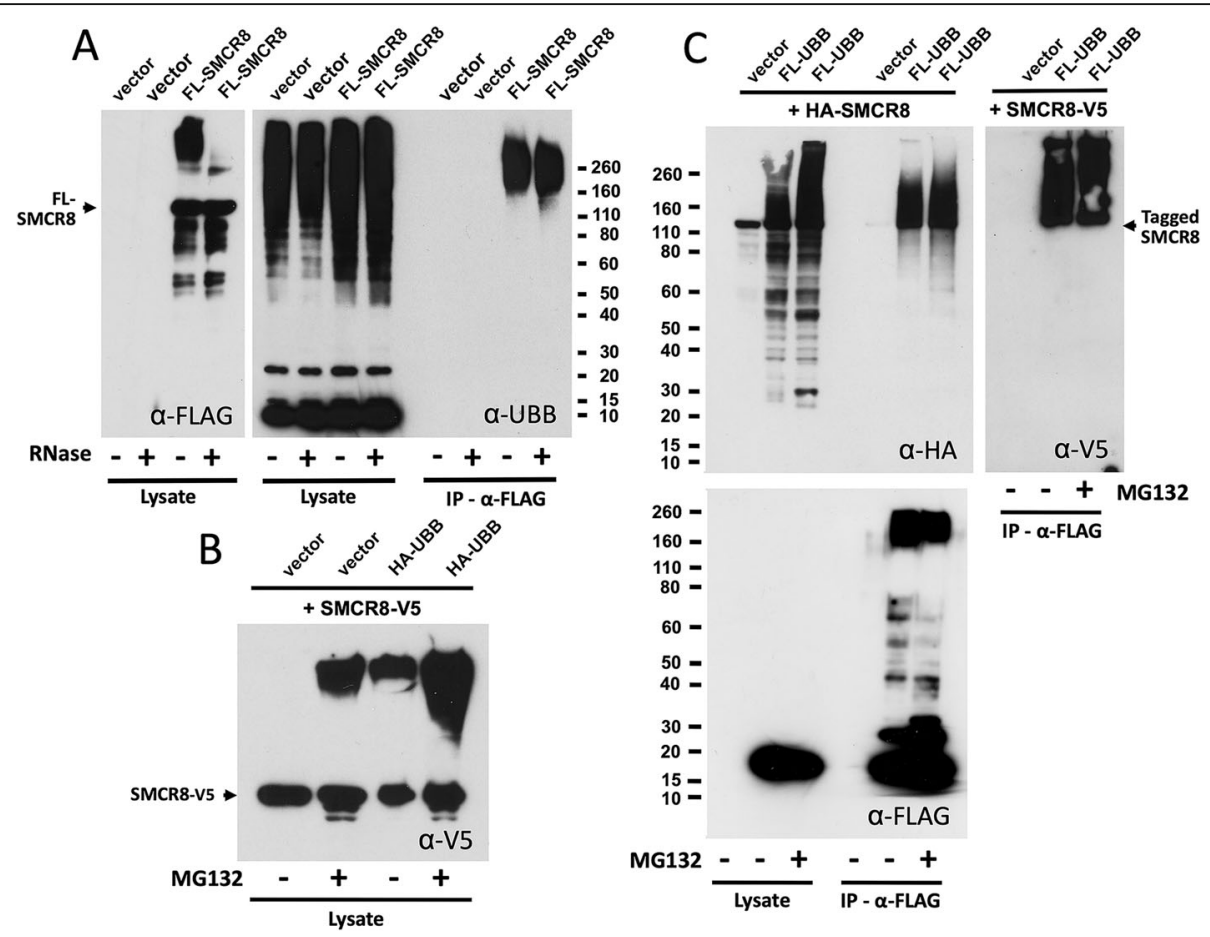

Fig. 3 Evidence that SMCR8 protein is poly-ubiquitinated. a The FL-SMCR8 construct was transfected in 293T cells and immunoprecipitated with a-FLAG antibody-bound agarose. A Western blot of whole cell lysates probed with a-FLAG antibody shows expression of full-length FL-SMCR8 protein plus HMW products consistent with PTMs (left). Probing with a-UBB antibody marks HMW products in immunoprecipitates consistent with either poly-ubiquitinated FL-SMCR8 protein or the presence of other HMW ubiquitinated proteins that co-IP with the SMCR8 complex (right). IP reactions were in the presence or absence of $50 \mu \mathrm{g} / \mathrm{ml}$ RNases. b C-terminal V5-tagged SMCR8 and empty vector or HA-tagged ubiquitin were coexpressed in $293 \mathrm{~T}$ cells and treated or not treated with the proteasome inhibitor MG132. Expression of SMCR8-V5 protein and empty vector, in the presence but not absence of MG132, produces HMW bands on Western blots that are consistent with post-translational modification of SMCR8 at multiple sites. SMCR8-V5 protein coexpressed with HA-UBB and without MG132 shows the same HMW bands, which increase in signal intensity upon incubation with MG132. c V5- or HA-epitope-tagged SMCR8 was coexpressed with empty vector or FLAG-tagged UBB in 293T cells and incubated overnight in the presence or absence of MG132. Cell lysates were subjected to immunoprecipitation with a-FLAG agarose,

followed by Western blotting and probing with a-HA (top left panel), a-V5 (top right) or a-FLAG (bottom left) antibodies. A HMW smear seen in immunoprecipitates is consistent with poly-ubiquitination of tagged SMCR8 proteins. In general, overexpression of ubiquitin does not lead to a significant decrease in full-length SMCR8 protein levels

associated cellular proteins, numbers of which have been linked with neuropathologies (Table 2).

\section{Evidence that endogenous SMCR8 accumulates in cytoplasmic stress granules}

The accumulation of neuronal RNA and protein aggregates, including cytoplasmic stress granules, is a pathogenic hallmark of a number of neurodegenerative diseases, among them FTD and ALS [93-95]. SGs assemble rapidly under cellular stress and include the small, but not large, ribosomal subunits bound to translation initiation factors such as eIF2 and eIF3 (reviewed in [96]). Processing-bodies (PBs) and SGs are dynamic cytoplasmic aggregates that participate in mRNA decay, and SGs in mammalian cells are heavily ubiquitinated [97]. Because previous publications implicated C9orf72 protein expression in the metabolism of SGs [88, 98], we wished to determine if the
C9orf72 binding partner SMCR8 associates with SGs in various tumor cell lines.

As reported by others [28], we observed epitope-tagged SMCR8 and C9orf72 proteins to both have a diffuse cytoplasmic distribution with protein also observed in nuclei, although nuclear localization was more evident for C9orf72 (Fig. 4b,c, S4A-C). However, although Maharjan et al. [98] reported that SGs were induced in a majority of unstressed mouse Neuro2A (N2A) neuroblastoma cells when transfected with myc-tagged C9-L, we failed to observe this phenomenon for tagged C9-L or SMCR8 proteins transfected alone or in combination (not shown) in unstressed human osterosarcoma U2OS, HEK 293T, or neuroblastoma cell lines (Fig. 4b, S4B). Furthermore, when cells were treated with $250 \mu \mathrm{M}$ of the oxidative stressor sodium arsenite $\left(\mathrm{NaAsO}_{2}\right)$ for $80 \mathrm{~min}$, tagged C9orf72, SMCR8, or WDR41 protein very rarely colocalized in aggregates with endogenous canonical SG marker protein TIA1 in multiple cell lines (Figs. 4c,d, S4C). 
A
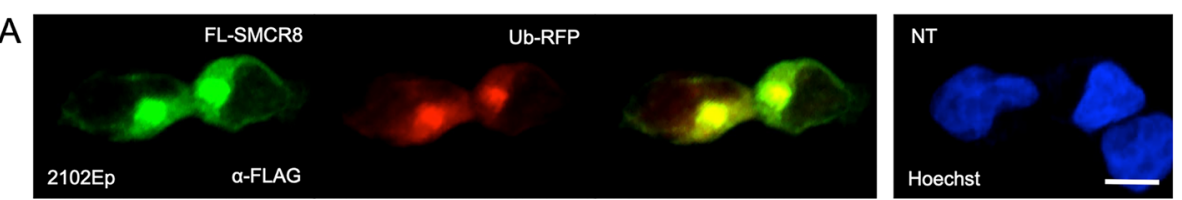

B

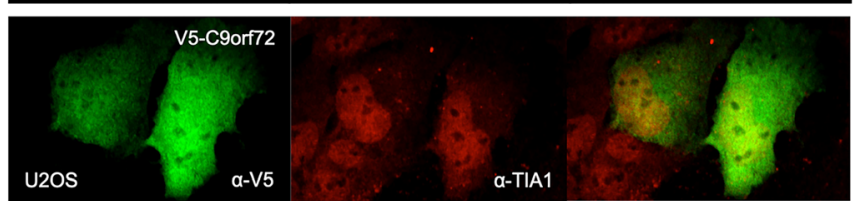

c

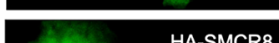

a-TIA1
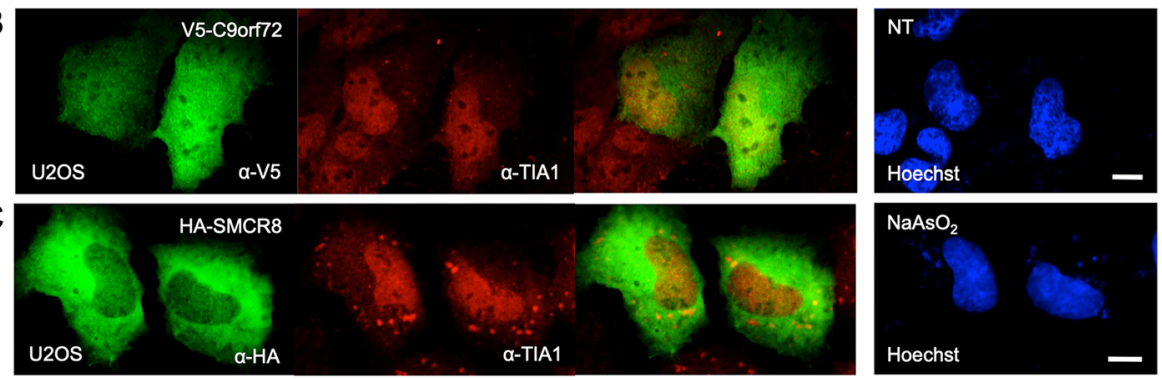

D

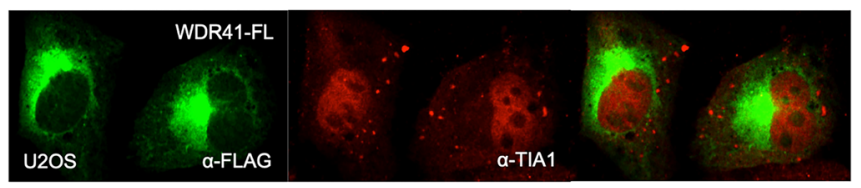

$\mathrm{E}$

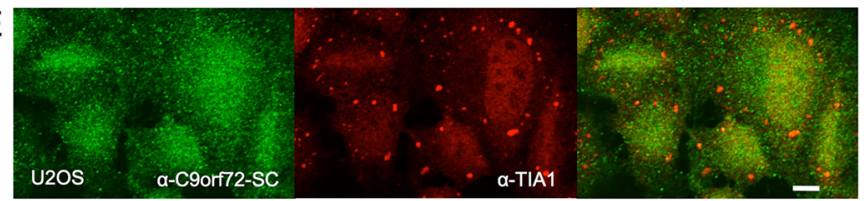

$\mathrm{F}$

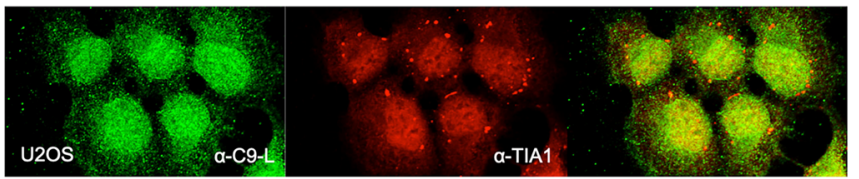

G

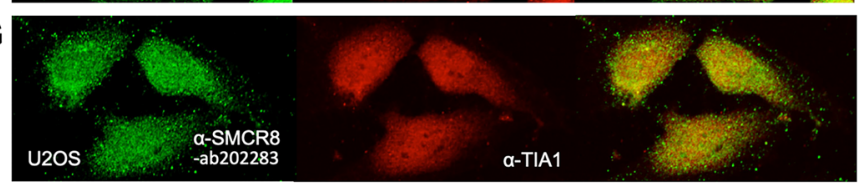

$\mathrm{H}$
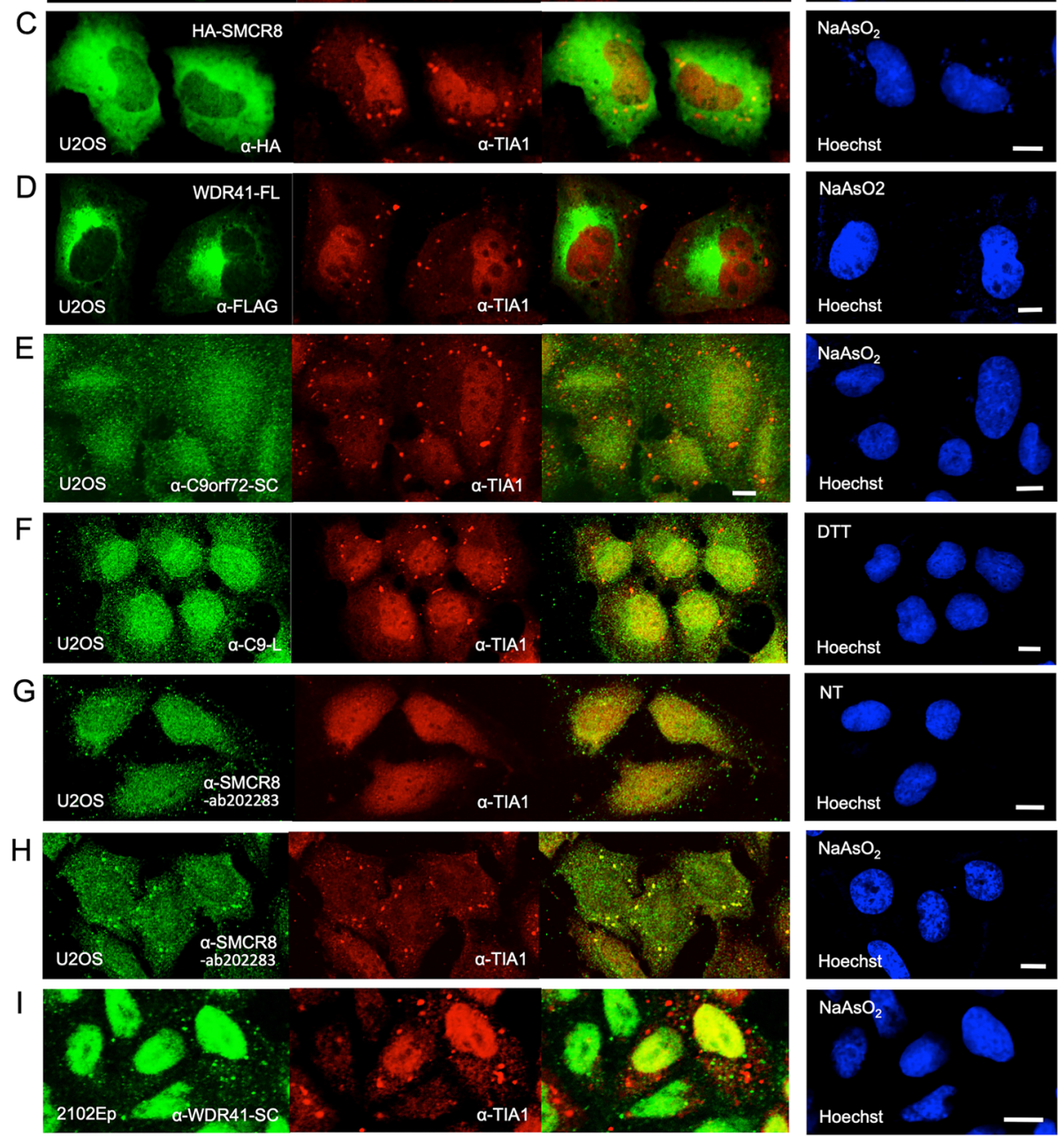

Fig. 4 Immunofluorescence microscopy shows evidence for association of endogenous SMCR8 protein with cytoplasmic aggregates. a FLAGtagged SMCR8 and RFP-tagged ubiquitin transfected in 2102Ep cells colocalize in a structure consistent with the aggresome. b Overexpression of V5-tagged C9orf72 does not induce stress granule formation in unstressed U2OS cells. c Exogenously expressed HA-SMCR8 protein is not observed in $\mathrm{SG}$ of U2OS cells stressed with $\mathrm{NaAsO}_{2}$. $\mathbf{d}$ WDR41-FL protein does not colocalize with SG marker protein TIA1 in U2OS cells stressed with $\mathrm{NaAsO}_{2}$. e Endogenous C9orf72 protein detercted by the a-SMCR8-SC antibody does not colocalize with SGs in $\mathrm{NaAsO}_{2}$-stressed U2OS cells (see also Fig. S4E). f Endogenous C9orf72 protein detected by the C9-L antibody [54] does not colocalize with SGs in DTT-stressed U2OS cells. g,h Endogenous SMCR8 detected by the a-SMCR8-ab202283 antibody localizes to SGs of stressed (h), but not unstressed (g) U2OS cells (see also Fig. S4G-I). i The a-WDR41-SC antibody does not detect endogenous protein in SGs of $\mathrm{NaAsO}_{2}$-stressed 2102Ep cells. NT: no treatment. Cell nuclei were stained with Hoechst 33342 (right-most panels). Size bars are $10 \mu \mathrm{m}$

We next examined localization of endogenous C9orf72 and SMCR8 proteins in cells. The $\alpha$-C9orf72-SC and $\alpha$ C9orf72-PT antibodies both detected nuclear and cytoplasmic distribution for C9orf72 protein, with fine cytoplasmic granululazion visible in unstressed cells that was more evident for the latter antibody (Fig. S4D). 
However, contrary to previous studies that used these antibodies to report SG localization [88, 98], we failed to detect endogenous C9orf72 in stress-induced U2OS (Figs. 4e, S4E) or 2102Ep (not shown) cells, although C9orf72 infrequently justaposed or overlapped with SGs and/or PBs in N2A cells (Fig. S4F.G). To confirm further these observations, two polyclonal antibodies developed by the Robertson lab [19, 79], and specific for C9-L (Fig. 4f) and C9-S (not shown) isoforms, were also tested but failed to show obvious C9orf72 protein presence in TIA1-marked SGs in DTT- or $\mathrm{NaAsO}_{2}$-stressed cells of multiple lines, including U2OS and 2012Ep cells. Thus, detection of C9orf72 in SGs appears to be cell line and possibly antibody dependent.

We also used the $\alpha$-SMCR8-PT and $\alpha$-SMCR8ab202283 antibodies to examine endogenous SMCR8 protein localization. In unstressed U2OS cells, endogenous SMCR8 was nuclear and more prominently cytoplasmic with speckled staining (Fig. 4g). However, when cells were stressed with $\mathrm{NaAsO}_{2}, \mathrm{SMCR} 8$ redistributed to large intensely staining foci that colocalized with TIA1 (Fig. 4h). Fig. S4H shows SMCR8 protein in large cytoplasmic aggregates of $\mathrm{NaAsO}_{2}$-stressed HEK 293T cells that costain with a different endogenous SG marker, the LINE-1 retrotransposon-encoded ORF1 protein [81], while Fig. S4I shows costaining with SGmarker eIF3 $\eta$ in human neuroblastoma SK-N-SH cells. In N2A cells treated with the endoplasmic reticulum stressor thapsigargin, SMCR8 granules were marked by a p70 S6 kinase antibody known to recognize HEDLS/ EDC4, a PB component (Fig. S4J, [77]): PBs frequently overlap or juxtapose with SGs in stressed cells [99]. Endogenous SMCR8 granules in unstressed N2A cells also partially colocalized with GW182 autoantigen, which marks PBs (Fig. S4K) [80]. However, as noted above, SMCR8 commercial antibodies detect multiple protein species (Fig. S1B-E), some possibly non-specific, and we cannot be certain that canonical full-length endogenous SMCR8 proteins are what we see in SGs. Nevertheless, our data suggest that in stressed cells a fraction of endogenous SMCR8 protein is directed to cytoplasmic SGs.

Our analyses showed that TAR DNA binding protein 43 (TDP-43, product of the TARDBP gene) binds SMCR8 (Fig. 2; Table 2). Mutations in TARDBP are involved in about $4 \%$ of familial and $1 \%$ of sporadic ALS (sALS) cases. However, even wild-type TDP-43, while mostly nuclear in healthy cells, is cleaved and hyperphosphorylated and accumulates in ubiquitinated cytoplasmic aggregates in neurons of almost all ALS and about half of FTLD patients (reviewed in [100]). We tested if endogenous or overexpressed SMCR8 protein colocalizes with TDP-43 protein in cytoplasmic granules but found this not to be the case in unstressed or stressed U2OS or 2102Ep cells (Fig. S4L).
Hexanucleotide expansions within transcripts of the C9orf72 ALS gene may undergo non-conventional repeat-associated non-ATG (RAN) translation and generate dipeptide repeats that aggregate in the cytoplasm of neuronal cells of C9ALS patients (reviewed in [101]). To see if such aggregates might colocalize with SMCR8, we coexpressed in 293T cells FL-SMCR8 and a C9orf72 RAN translation product of 50 GA-dipeptide repeats tagged with EGFP [68]. Overexpressed dipeptide proteins formed one to three large cytoplasmic aggrgates in each cell that were were ringed by, but mostly excluded SMCR8 (Fig. S4M).

Finally, the $\alpha$-WDR41-SC antibody marks WDR41 protein as predominantly nuclear but also with faint cytoplasmic granules that fail to colocalize with SGs in unstressed or stressed U2OS, 2102Ep, 293T, or N2A cells (Fig. 4i and not shown). On the other hand, the $\alpha$ WDR41-PT antibody colocalizes with a minor subset of granules positive for 4-ET, a marker of PBs (Fig. S4N). However, while the $\alpha$-WDR41-SC antibody recognizes only bands consistent in size with WDR41 isoforms in HEK 293T, 2102Ep, and SK-N-SH cells (Fig. S1F), the $\alpha$ WDR41-PT antibody detects other non-canonical protein species (Fig. S1G), and the specificity of its SG staining is thus uncertain.

Searching the Mammalian Stress Granules Proteome Database (https://msgp.pt) [102], we found that $18 \%$ of the SMCR8 proiein interactome (61/340) and 26\% (35/ 201) of the C9orf72 interactome are known SGassociated proteins. It is thus possible that SG components bind endogenous SMCR8-C9orf72 complexes and shepherd them to SGs, although why this would not also be the case for overexpressed exogenous SMCR8 or C9orf72 proteins is unclear.

\section{SMCR8 expression in ALS patient brain tissues}

Despite its strong association with protein-degradation factors, SMCR8 overexpression does not stimulate degradation of $\mathrm{C} 9$ orf72 protein with which it is in complex. Contrarily, multiple studies in cells and knockout mice have shown that protein but not RNA levels of SMCR8 and $C 9$ orf 72 are positively correlated, suggesting that in complex the two proteins stabilize and protect each other from degradation [26, 28, 29, 32, 47, 54, 65, 103]. On the other hand, increased SMCR8 protein reportedly has little effect on WDR41 levels in KO mice or cells [32, 35]. We confirmed in $293 \mathrm{~T}$ cells that overexpression of SMCR8 with various tags strongly increased levels of cotransfected FL-C9-L protein, while cotransfection of empty vectors or an unrelated protein (RO60) did not (Fig. 5a). Considering the interplay between SMCR8 and C9orf72 proteins, and the fact that C9orf72 RNA expression is reduced in some C9ALS patient cohorts, we asked if SMCR8 expression levels are altered in the 

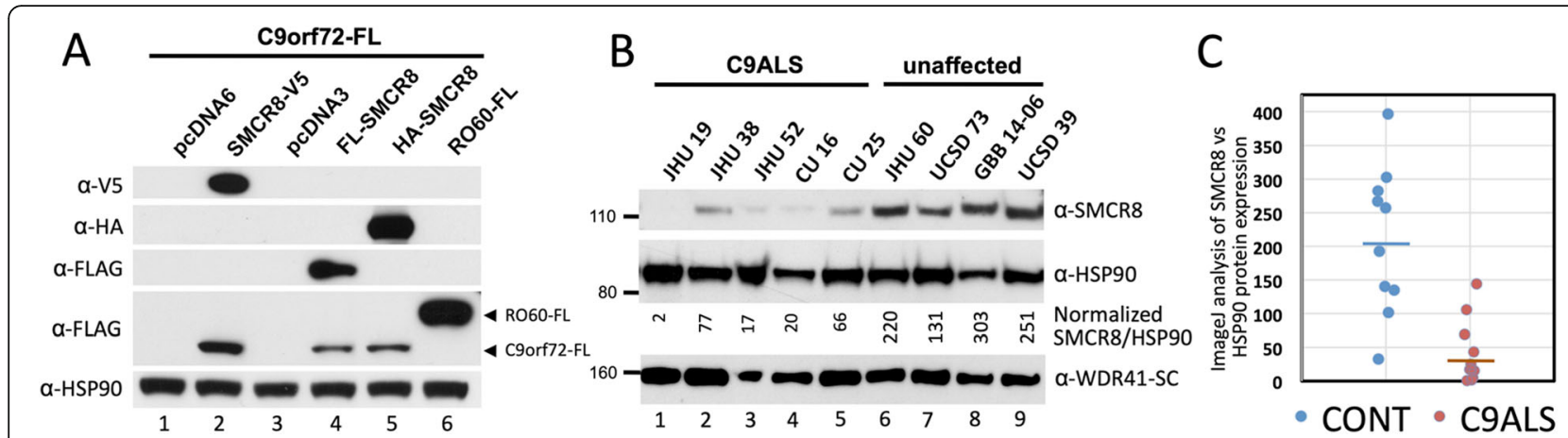

Fig. 5 Expression of C9orf72 and SMCR8 proteins are positively correlated in cell lines and human brain tissues. a C9orf72-FL was coexpressed in HEK 293 T cells with 3 different epitope-tagged SMCR8 constructs, FLAG-tagged RO60 protein, or empty vectors (pcDNA3 and pcDNA6 myc/his B). A Western blot of whole cell lysates was probed sequentially with rb a-FLAG, ms a-HA, ms a-V5, and rb a-HSP90 antibodies, the latter as a loading control. At the exposure time for the film shown, expression of C9orf72-FL was not seen in the presence of empty vector or RO60-FL, but signal was robust in the presence of SMCR8. b Western blot of brain motor cortex tissue lysates of C9ALS patients (lanes 1-5) and unaffected control individuals (lanes 6-9) probed with a-SMCR8 and a-HSP90 antibodies. Sample names are shown above the panels (see Table S4). Numbers below the middle panel are normalized ratios of SMCR8 to HSP90 expression determined by ImageJ analysis of band intensities and calculated as described in the text. The lower panel shows the approximately 150-kD unspecified band detected by a-WDR41-SC antibody in human brain tissue lysates (see Fig. S1F): this panel is included only as an additional loading control and is not intended to show expression of canonical WDR41 protein. Approximtely $50 \mathrm{\mu g}$ of protein was loaded in each lane. c Dot plot of ratios of SMCR8 to HSP90 protein band intensities determined by ImageJ analyses of brain tissues lysates from 11 C9ALS and 10 control individuals. Each sample point is the average of 2 to 4 independent Western blot analyses. A short horizontal line indicates mean values. The presence of a C9orf72 hexanucleotide expansion in each C9ALS carrier individual was confirmed by Columbia University and Target ALS using RP-PCR and Illumina Expansion Hunter, but expansion copy numbers are not known

brains of C9ALS patients compared with non-affected controls.

We first examined transcription levels of C9orf72, SMCR8, and WDR41 genes in RNA-Seq datasets from several sequence read archives that contain C9ALS sample data. GEO dataset GSE67196 includes cerebellum and frontal cortex samples of 9 healthy, 8 C9ALS, and 10 sALS individuals. Using TEtranscripts [84] to analyze C9orf72 gene expression levels, we found a significant $\log _{2}$ 0.96-fold decrease (padj 4.6E-5) in the frontal cortex of C9ALS vs sALS individuals and a 1.1-fold decrease (padj 1.6-E4) in the cerebellum of C9ALS vs control individuals; however, in neither case was decrease in SMCR8 expression significant. The NeuroLINCS dbGaP Study phs001231 (SRP098831) consists of poly(A) + non-stranded mRNA of iPSC-derived motor neurons from 4 C9ALS, 3 spinal muscular atrophy (SMA), and 3 unaffected individuals ( 2 or 3 replicates each). No significant changes in C9orf72 or SMCR8 transcript levels were seen in this dataset, although WDR41 sequence read numbers were reduced about 0.35 -fold in both C9ALS vs control and SMA vs control samples $($ padj<0.01). Finally, a recent RNA-Seq study comparing C9 FTLD and FTLD/ motor neuron disease patients with unaffected control individuals reported a highly significant decrease in C9orf72 RNA levels in C9 FTLD samples; however, this data showed no significant change in SMCR8 or WDR41 RNA expression [24].
We next assayed endogenous SMCR8 protein expression levels in the context of the C9orf72 hexanucleotide expansion. Motor cortex brain tissue lysate samples of 11 C9ALS and 10 unaffected control individuals were analyzed by Western blotting with $\alpha$-SMCR8 antibodies (Fig. 5b, Table S4). Multiple film exposures were made to optimize signal to noise. Individual band intensities were quantitated with Image software [104] and normalized against the summed exposures of all equivalent bands on the same gel. SMCR8 signal was then normalized to endogenous HSP90 protein signal detected on the same gel after reprobing with $\alpha$-HSP90 antibody. Remarkably, an average 5-fold reduction in SMCR8 protein signal was seen in C9ALS vs control tissues (Fig. 5b). We also tested by Western blotting cerebrospinal fluid samples from 5 C9ALS patients and 5 unaffected controls, but were unable to detect full-length SMCR8 protein signal with either the $\alpha$-SMCR8-PT or $\alpha$-SMCR8ab202283 antibodies (not shown). We also plotted normalized SMCR8 protein signal against ALS disease duration in months (Table S4), finding a weak negative but non-significant correlation $(r=0.34)$. Nevertheless, altogether our data recommend further investigation of SMCR8 protein level as a potential biomarker of the C9orf72 expansion disease mutation.

\section{Discussion}

In this study we characterized the SMCR8 protein interactome and found it to include numerous components 
of the ubiquitin-proteasome system, including ubiquitin ligases and peptidases. Of note, the IP method used here exploited FLAG-tagged proteins and so overcame limitations imposed by differences in isoform expression and non-specific protein species recognized by C9orf72 and SMCR8 antibodies. Despite evidence that SMCR8 itself is ubiquitinated at multiple residues, its degradation is not significantly induced in the presence of overexpressed ubiquitin suggesting other roles linking it with the UPS. Recruitment of UPS components to autophagy complexes could be one such role, and our SMCR8 interactome contains 24 autophagy pathway-associated proteins (Table 1). Ubiquitin plays a fundamental role not only in proteasome-mediated protein degradation but also in the targeting of proteins for degradation by autophagic complexes. Protein ubiquitination also regulates multiple steps of the autophagy pathway (reviewed in $[105,106])$. For example, E3 ubiquitin ligase STUB1, a protein that co-IPs with SMCR8 (Table 1, Fig. 2), regulates autophagy by targeting TFEB for degradation by the UPS [107]. Also, E3 ligase HUWE1 (Table 1) mediates the ubiquitination and proteasomal degradation of WIPI2, a protein involved in autophagosome formation [108].

Recruitment of ubiquitination factors would be consistent with reported roles of the C9orf72-SMCR8 complex in autophagy and endosomal-lysosomal metabolism. The C9orf72/SMCR8/WDR41 complex associates with the ATG8 autophagy receptor network and influences activity of the ULK1-RB1CC1-ATG13ATG101 autophagy initiation complex (RB1CC1 was detected in both our SMCR8 and C9orf72 protein interactomes, Tables S1, S2) [22, 28, 29, 31, 34]. C9orf72 and SMCR8 reportedly also play roles in regulating mTORC1 and TFEB autophagy and lysosomal gene transcription factors upstream of autophagy [26, 31, 32, $34,57,103,109,110]$. Ugolino et al. [32], however, indicated a negative effect of C9orf72 on autophagy, and Yang et al. [31] reported increased autophagic flux in C9orf72 knockdown MEF cells, opposite to the reduction they saw in SMCR8-deficient cells. Unlike some C9orf72-deficient mice [45], Smcr8 KO mice showed motor behavior defects, including axonal swelling due to impaired autophagy and motor neuron axonal transport of autophagosomes [25]. Apparently, although in complex, some biological roles of C9orf72 and SMCR8 proteins differ.

Association of the SMCR8-C9orf72 complex with the UPS and autophagy would also be consistent with stress granule localization, since protein ubiquitination regulates SG dynamics. Components of the UPS, including ubiquitin, co-localize with SGs, while proteasome inhibition, and consequent increase in ubiquitinated proteins, induces SG formation [111-113]. Recent evidence also suggests SGs are regulated by autophagy [114, 115], and it has been proposed that improper metabolism of SGs could be involved in ALS pathology [93, 94]. Interestingly, Chitiproulu et al. [88] proposed that C9orf72 protein associates with autophagy cargo receptor p62 (encoded by the SQSTM1 gene) to control SG elimination rather than assembly by forming a complex that eliminates by autophagy SG proteins dimethylated on arginines (of note, we found p62 in the SMCR8 but not C9orf72 interactomes; Fig. 2, Table 2S).

However, our data disagree in some aspects with previously published results concerning C9orf72 colocalization with SGs. While, Maharjan et al. [98] reported that overexpression of myc-tagged C9-L led to the spontaneous appearance of SGs in a majority of N2A cells and cortical neurons in the absence of cellular stress, we failed to reproduce these observations in either U2OS or N2A cells for tagged C9orf72 or SMCR8 proteins, overexpressed together or separately. Furthermore, using the $\alpha$-C9orf72-PT antibody (Fig. S1A), Maharjan et al. [98] noted that endogenous C9orf72 protein colocalized with a fraction of SGs in neuronal cell lines and cortical neurons in response to DTT and heat shock-induced cell stress, and that C9orf72 depletion inhibited SG assembly, impaired expression of proteins required for their formation, and increased cell sensitivity to stress. However, despite testing several antibodies, cell lines and conditions, we could not detect endogenous C9orf72 in SGs of selected non-neuronal cancer-derived cell lines, and we saw only minor colocalization of C9orf72 with SGs and PBs of N2A cells. Thus, association of C9orf72 protein to SGs appears to be cell line-dependent.

On the other hand, we observed endogenous, but not exogenously expressed, SMCR8 protein localization to SGs of all chemically stressed cell lines tested. Interestingly, about one-fifth of the putative interacting proteins we identified as members of our C9orf72 and SMCR8 interactomes are known SG proteins, which themselves might play a role in targeting of SMCR8 complexes to granules. It is conceivable that SMCR8-C9orf72 SG association is sensitive to cell type, cellular conditions, and levels of interacting proteins as determinants of entry into SGs, and perhaps these factors explain discrepancies between our data and previously published observations.

As reported in other studies, we also presented supporting evidence that C9orf72 protein levels are positively correlated with those of SMCR8 in cultured cells $[26,28,29,32,47,54,65,103]$. Furthermore, we now show that SMCR8 protein expression is reduced in the brains of C9ALS patients compared with unaffected controls (and as also recently noted by [25]. To date, it has been reported that a small number of proteins, including neurofilament proteins, are differentially expressed in the CSF of ALS and FTD proteins and have been 
proposed as candidate biomarkers for the C9orf72 mutation $[116,117]$. Whether or not SMCR8 protein can also be an effective CSF or plasma biomarker for C9 expansion patients remains to be determined and is likely contingent upon the development of better $\alpha$-SMCR8 antibodies.

\section{Conclusions}

In this study we characterized the protein interactome of SMCR8, which binds the protein product of C9orf72, the major susceptibility gene for ALS. Using a robust and highly specific protocol, we demonstrated ubiquitination without significant degradation of SMCR8 protein and its association with many components of the ubiquitinproteasome system. Evidence was presented for localization of endogenous SMCR8 protein to cytoplasmic stress granules, although in several cell lines we failed to reproduce previous observations that C9orf72 protein enters these granules. SMCR8 protein levels were downregulated in whole tissue brain lysates of C9ALS patients compared with unaffected controls, suggesting the potential usefulness for SMCR8 as a biomarker of the disease state.

In addition to ALS and FTD, the C9orf72 gene expansion mutation has been linked with other neurodegenerative and psychiatric disorders, although etiological roles remain unknown [118-123]. We have shown that SMCR8, whose cellular levels positively correlate with C9orf72 protein expression, associates not only with many factors of protein metabolism and stress granule dynamics, but also with numerous products of genes linked with a range CNS disorders (65/340 in total, Table 2). It is therefore reasonable in future studies to consider a role for SMCR8 in these diverse neuropathologies, perhaps relating to recruitment of the UPS with consequent effects on protein homeostasis.

\section{Supplementary information}

Supplementary information accompanies this paper at https://doi.org/10. 1186/s40478-020-00982-x.

Additional file $\mathbf{1}$ Fig. S1. Western blotting of cell line and brain tissue lysates using commercial antibodies against C9orf72, SMCR8, and WDR41 proteins. Antibodies shown are (A) a-C9ORF72-PT (Proteintech Group 22637-1), (B) a-SMCR8-PT (Proteintech 21125-1-AP, (C) a-SMCR8-B (Bethyl Laboratories A304-694A), (D) a-SMCR8-ab186504 (Abcam ab186504), (E) a-SMCR8-ab202283 (Abcam ab202283), (F) a-WDR41-SC (Santa Cruz sC137923), and (G) a-WDR41-PT (Proteintech 26817-1-AP). Large arrows mark full-length protein products. The small arrows in (F) and (G) mark the presumed minor $45.5 \mathrm{kD}$ WDR41 isoform. Samples were run on NuPAGE 4-12\% Bis-Tris Protein gels (Novex) and Western blotting was performed according to [81, 82]. Molecular weight markers are Novex Sharp Pre-stained Protein Standard. Motor: motor cortex, Occ: occipital cortex, S.C.: spinal cord; sample identifier numbers are also shown. Fig. S2. Pie chart of results of DAVID (Database for Annotation, Visualization and Integrated Discovery, [83]) analyses of KEGG pathways showing selected functional categories for candidate member proteins of the (A) FLSMCR8 and (B) C9orf72-FL protein interactomes. Percentages of the total number of proteins identified (Tables S1 and S2) for each category are shown within the slices. Fig. S3. Phylogenetic multi-sequence alignment of SMCR8 protein sequences for ten species. Alignments were made with Clustal Omega 1.2.1 (EMBL-EBI) followed by BoxShade 3.2 (http://sourceforge.net/projects/boxshade). Pink shading marks amino acid residues identical in at least 8 species, while green includes conservative replacements. Lysine residues predicted by MS sequencing to be ubiquitinated are boxed in blue (see Table S3). Species shown are: Homo sapiens, human; Pan troglodytes, chimpanzee; Canus lupus familiaris, dog; Mus musculus, house mouse; Rattus norvegicus, brown rat; Gallus gallus domesticus, chicken; Danio rerio, zebrafish; Xenopus tropicalis, western clawed frog; Biomphalaria glabrata, freshwater snail; Aplysia californica, California sea hare. Fig. S4. Immunofluorescence microscopy evidence for association of SMCR8 protein with cytoplasmic aggregates. (A) V5-tagged SMCR8 does not significantly enter $\mathrm{SGs}$ of $\mathrm{NaAsO}_{2}$-stressed N2A cells. (B) Overexpression of FLAG-tagged C9orf72 (left) or SMCR8 (right) protein does not induce formation of cytoplasmic granules in neuroblastoma cells. (C) V5tagged exogenously expressed C9orf72 fails to enter $\mathrm{SG}_{5}$ in $\mathrm{NaAsO}_{2^{-}}$ treated U2OS cells. (D) Endogenous C9orf72 protein speckles observed in unstressed N2A cells are not marked by TIA1, a SG protein. (E) C9orf72 protein detected by the a-C9orf72-PT antibody fails to enter SGs of $\mathrm{NaAsO}_{2}$-stressed U2OS cells. $(F, G)$, Endogenous C9orf72 protein detected by the a-C9orf72-SC antibody colocalizes or justaposes with only a minor subset of SGs (marked by TIA1) or PBs (marked by 4-ET) in stressed mouse neuroblastoma N2A cells (see arrows). (H) In $\mathrm{NaAsO}_{2}$-stressed 293T cells, endogenous SMCR8 detected by the a-SMCR8-PT antibody colocalizes with granules marked by ORF1 $\mathrm{p}$, a protein encoded by LINE-1 retrotransposons [81]. (I) In $\mathrm{NaAsO}_{2}$-stressed human neuroblastoma SK-NSH cells, endogenous SMCR8 localizes to SGs marked by a-elF3n antibody. (J) In N2A cells treated with the endoplasmic reticulum stressor thapsigarin, endogenous SMCR8 protein colocalizes to granules marked by HEDLS/EDC4 (detected by a-p70 56 kinase antibody, [77]), a component of PBs. (K) In unstressed N2A cells, endogenous SMCR8 localizes in granules with GW142, a PB marker. (L) Exogenously expressed TDP-43 and endogenous SMCR8 proteins do not colocalize in cytoplasmic granules of stressed U2OS cells. (M) SMCR8 rings but is generally excluded from overexpressed GFP-(GA) 50 dipeptide aggregates. (M) In 293T cells, the a-WDR41-PT antibody detects endogenous protein in or beside a subset granules marked by 4-ET (see arrows). NT: no treatment. Cell nuclei were stained with Hoechst 33342 (right-most panels). Size bars are $10 \mu \mathrm{m}$. Table S1. The C9orf72 protein interactome determined by mass spectrometry. Table S2. The SMCR8 protein interactome determined by mass spectrometry. Table S3. Ubiquitinated SMCR8 lysine residues determined by MS sequencing, prediction algorithms, and the comparative phylogenetic analyses of Fig. S3. Table S4. Post-mortem brain motor cortex tissue samples used for the analyses of Fig. 5b,c.

\section{Abbreviations}

ALS: Amyotrophic lateral sclerosis; C9ALS: C9orf72 repeat expansion ALS; CNS: Central nervous system; CSF: Cerebrospinal fluid; FTD: Frontotemporal dementia; FTLD: Frontotemporal lobar degeneration; GEF: Guanine nucleotide exchange factor; GFP: Green fluorescent protein; HMW: High molecular weight; IF: Immunofluorescence; IP: Immunoprecipitation; iPSC: Induced pluripotent stem cell; kD: Kilodalton; N2A: Neuro2A; ORF: Open reading frame; PB: Processing (P-) body; PCR: Polymerase chain reaction; PTM: Post-translational modification; RFP: Red fluorescent protein; sALS: Sporadic ALS; SG: Stress granule; UPS: Ubiquitin-proteasome system

\section{Acknowledgements}

The authors appreciate reagents provided by researchers listed in the Materials and Methods section. Human samples were received from the Target ALS Multicenter Postmortem Tissue Core (with thanks to K. Wilsbach and Dr. L. Ostrow), Drs. J. Ravits and R. Batra of the University of California San Diego, the University of Maryland Brain and Tissue Bank of the NIH NeuroBioBank, and the Northeast ALS Consortium (NEALS), as described above. Services and reagents were provided by the Johns Hopkins University School of Medicine ChemCore, Synthesis \& Sequencing Facility, and Mass Spectrometry and Proteomics Core. Special thanks to Dr. Haig H. Kazazian and his laboratory (Johns Hopkins University School of Medicine) for ongoing advice and support. 


\section{Authors' contributions}

JLG designed experiments and wrote the manuscript. AS, GCP, JLG, JLGP and LS performed experiments. AS and JLGP assisted with manuscript editing. RNC and LRD performed MS analyses and assisted with data interpretation and manuscript review. The author (s) read and approved the final manuscript.

\section{Funding}

JLG was supported by grants from the NIH National Institute of Neurological Disorders and Stroke (1R03NS087290-01), the NIH Eunice Kennedy Shriver National Institute of Child Health and Human Development (R21HD08391501A1), and the ALS Therapy Alliance (2013-F-067). The JLGP lab acknowledges funding from the European Research Council (ERCConsolidator ERC-STG-2012-309433), the MINECO-FEDER (SAF2017-89745-R), and The Wellcome Trust-University of Edinburgh Institutional Strategic Support Fund (ISFF2). RNC was partially supported by NIH/NCATS grant UL1 TR003098.

\section{Availability of data and materials}

Raw MS sequencing data used to generate Supplementary Tables S1 and S2 will be made available from the corresponding author upon request.

\section{Ethics approval and consent to participate}

All post-mortem tissues were obtained following approval of the Institutional Review Board of the JHU School of Medicine (IRB00066246 to JLG).

\section{Consent for publication}

Not applicable.

\section{Competing interests}

The authors declare that they have no competing interests.

\begin{abstract}
Author details
${ }^{1}$ McKusick-Nathans Department of Genetic Medicine, Johns Hopkins University School of Medicine, Baltimore, MD, USA. ${ }^{2}$ Mass Spectrometry and Proteomics Facility, Department of Biological Chemistry, Johns Hopkins University School of Medicine, Baltimore, MD, USA. ${ }^{3}$ GENYO. Centre for Genomics and Oncological Research: Pfizer, University of Granada, Andalusian Regional Government, Granada, Spain. ${ }^{4}$ MRC Human Genetics Unit, Institute of Genetics and Molecular Medicine (IGMM), University of Edinburgh, Western General Hospital, Edinburgh, UK.
\end{abstract}

Received: 17 April 2020 Accepted: 26 June 2020

\section{Published online: 16 July 2020}

\section{References}

1. Brown RH, Al-Chalabi A (2017) Amyotrophic lateral sclerosis. N Engl J Med 377(2):162-172

2. Miller B, Llibre Guerra JJ (2019) Frontotemporal dementia. Handb Clin Neurol 165:33-45

3. Abramzon YA, Fratta P, Traynor BJ, Chia R (2020) The overlapping genetics of amyotrophic lateral sclerosis and frontotemporal dementia. Front Neurosci 14:42

4. DeJesus-Hernandez M, Mackenzie IR, Boeve BF, Boxer AL, Baker M, Rutherford NJ et al (2011) Expanded GGGGCC hexanucleotide repeat in noncoding region of C9ORF72 causes chromosome 9p-linked FTD and ALS. Neuron 72(2):245-256

5. Renton AE, Majounie E, Waite A, Simon-Sanchez J, Rollinson S, Gibbs JR et al (2011) A hexanucleotide repeat expansion in C9ORF72 is the cause of chromosome 9p21-linked ALS-FTD. Neuron 72(2):257-268

6. Gijselinck I, Van Langenhove T, van der Zee J, Sleegers K, Philtjens S, Kleinberger $\mathrm{G}$ et al (2012) A C9orf72 promoter repeat expansion in a Flanders-Belgian cohort with disorders of the frontotemporal lobar degeneration-amyotrophic lateral sclerosis spectrum: a gene identification study. Lancet Neurol 11(1):54-65

7. Todd TW, Petrucelli L (2016) Insights into the pathogenic mechanisms of chromosome 9 open reading frame 72 (C9orf72) repeat expansions. J Neurochem 138(Suppl 1):145-162

8. Ji AL, Zhang X, Chen WW, Huang WJ (2017) Genetics insight into the amyotrophic lateral sclerosis/frontotemporal dementia spectrum. J Med Genet 54(3):145-154
9. Balendra R, Isaacs AM (2018) C9orf72-mediated ALS and FTD: multiple pathways to disease. Nat Rev Neurol 14(9):544-558

10. Babic Leko M, Zupunski V, Kirincich J, Smilovic D, Hortobagyi T, Hof PR et al (2019) Molecular mechanisms of Neurodegeneration related to C9orf72 Hexanucleotide repeat expansion. Behav Neurol 2019:2909168

11. Almeida S, Gascon E, Tran H, Chou HJ, Gendron TF, Degroot S et al (2013) Modeling key pathological features of frontotemporal dementia with C9ORF72 repeat expansion in IPSC-derived human neurons. Acta Neuropathol 126(3):385-399

12. Mori K, Weng SM, Arzberger T, May S, Rentzsch K, Kremmer E et al (2013) The C9orf72 GGGGCC repeat is translated into aggregating dipeptide-repeat proteins in FTLD/ALS. Science 339(6125):1335-1338

13. Donnelly CJ, Zhang PW, Pham JT, Haeusler AR, Mistry NA, Vidensky $S$ et al (2013) RNA toxicity from the ALS/FTD C9ORF72 expansion is mitigated by antisense intervention. Neuron 80(2):415-428

14. Belzil W, Bauer PO, Prudencio M, Gendron TF, Stetler CT, Yan IK et al (2013) Reduced C9orf72 gene expression in C9FTD/ALS is caused by histone trimethylation, an epigenetic event detectable in blood. Acta Neuropathol 126(6):895-905

15. Fratta P, Poulter M, Lashley T, Rohrer JD, Polke JM, Beck J et al (2013) Homozygosity for the C9orf72 GGGGCC repeat expansion in frontotemporal dementia. Acta Neuropathol 126(3):401-409

16. Xi Z, Zinman L, Moreno D, Schymick J, Liang Y, Sato C et al (2013) Hypermethylation of the CpG island near the G4C2 repeat in ALS with a C9orf72 expansion. Am J Hum Genet 92(6):981-989

17. Waite AJ, Baumer D, East S, Neal J, Morris HR, Ansorge O et al (2014) Reduced C9orf72 protein levels in frontal cortex of amyotrophic lateral sclerosis and frontotemporal degeneration brain with the C9ORF72 hexanucleotide repeat expansion. Neurobiol Aging 35(7):1779.e5-1779e13

18. van Blitterswijk M, Gendron TF, Baker MC, DeJesus-Hernandez M, Finch NA, Brown PH et al (2015) Novel clinical associations with specific C9ORF72 transcripts in patients with repeat expansions in C9ORF72. Acta Neuropathol 130(6):863-876

19. Xiao S, MacNair L, McGoldrick P, McKeever PM, McLean JR, Zhang M et al (2015) Isoform-specific antibodies reveal distinct subcellular localizations of C9orf72 in amyotrophic lateral sclerosis. Ann Neurol 78(4):568-583

20. Rizzu P, Blauwendraat C, Heetveld S, Lynes EM, Castillo-Lizardo M, Dhingra A et al (2016) C9orf72 is differentially expressed in the central nervous system and myeloid cells and consistently reduced in C9orf72, MAPT and GRN mutation carriers. Acta Neuropathol Commun 4(1):37

21. Shi Y, Lin S, Staats KA, Li Y, Chang WH, Hung ST et al (2018) Haploinsufficiency leads to neurodegeneration in C9ORF72 ALS/FTD human induced motor neurons. Nat Med 24(3):313-325

22. Webster $\mathrm{CP}$, Smith EF, Bauer CS, Moller A, Hautbergue GM, Ferraiuolo L et al (2016) The C9orf72 protein interacts with Rab1a and the ULK1 complex to regulate initiation of autophagy. EMBO J 35(15):1656-1676

23. Frick P, Sellier C, Mackenzie IRA, Cheng CY, Tahraoui-Bories J, Martinat C et al (2018) Novel antibodies reveal presynaptic localization of C9orf72 protein and reduced protein levels in C9orf72 mutation carriers. Acta Neuropathol Commun. 6(1):72

24. Dickson DW, Baker MC, Jackson JL, DeJesus-Hernandez M, Finch NA, Tian S et al (2019) Extensive transcriptomic study emphasizes importance of vesicular transport in C9orf72 expansion carriers. Acta Neuropathol Commun. 7(1):150

25. Liang C, Shao Q, Zhang W, Yang M, Chang Q, Chen R et al (2019) Smcr8 deficiency disrupts axonal transport-dependent lysosomal function and promotes axonal swellings and gain of toxicity in C9ALS/FTD mouse models. Hum Mol Genet 28(23):3940-3953

26. Amick J, Roczniak-Ferguson A, Ferguson SM (2016) C9orf72 binds SMCR8, localizes to lysosomes, and regulates mTORC1 signaling. Mol Biol Cell 27(20):3040-3051

27. Ciura S, Sellier C, Campanari ML, Charlet-Berguerand N, Kabashi E (2016) The most prevalent genetic cause of ALS-FTD, C9orf72 synergizes the toxicity of ATXN2 intermediate polyglutamine repeats through the autophagy pathway. Autophagy. 12(8):1406-1408

28. Sullivan PM, Zhou X, Robins AM, Paushter DH, Kim D, Smolka MB et al (2016) The ALS/FTLD associated protein C9orf72 associates with SMCR8 and WDR41 to regulate the autophagy-lysosome pathway. Acta Neuropathol Commun. 4(1):51

29. Sellier C, Campanari ML, Julie Corbier C, Gaucherot A, Kolb-Cheynel I, OuladAbdelghani M et al (2016) Loss of C9ORF72 impairs autophagy and 
synergizes with polyQ Ataxin-2 to induce motor neuron dysfunction and cell death. EMBO J 35(12):1276-1297

30. Xiao S, MacNair L, McLean J, McGoldrick P, McKeever P, Soleimani S et al (1647) C9orf72 isoforms in amyotrophic lateral sclerosis and Frontotemporal lobar degeneration. Brain Res 2016:43-49

31. Yang M, Liang C, Swaminathan K, Herrlinger S, Lai F, Shiekhattar R et al (2016) A C9ORF72/SMCR8-containing complex regulates ULK1 and plays a dual role in autophagy. Sci Adv 2(9):e1601167

32. Ugolino J, Ji YJ, Conchina K, Chu J, Nirujogi RS, Pandey A et al (2016) Loss of C9orf72 enhances autophagic activity via deregulated mTOR and TFEB signaling. PLoS Genet 12(11):e1006443

33. Corbier C, Sellier C (2017) C9ORF72 is a GDP/GTP exchange factor for Rab8 and Rab39 and regulates autophagy. Small GTPases 8(3):181-186

34. Jung J, Nayak A, Schaeffer V, Starzetz T, Kirsch AK, Muller S et al (2017) Multiplex image-based autophagy RNAi screening identifies SMCR8 as ULK1 kinase activity and gene expression regulator. Elife 6:e23063

35. Amick J, Tharkeshwar AK, Amaya C, Ferguson SM (2018) WDR41 supports lysosomal response to changes in amino acid availability. Mol Biol Cell 29(18):2213-2227

36. Bi W, Yan J, Stankiewicz P, Park SS, Walz K, Boerkoel CF et al (2002) Genes in a refined Smith-Magenis syndrome critical deletion interval on chromosome 17p11.2 and the syntenic region of the mouse. Genome Res 12(5):713-728

37. Madduri N, Peters SU, Voigt RG, Llorente AM, Lupski JR, Potocki L (2006) Cognitive and adaptive behavior profiles in Smith-Magenis syndrome. J Dev Behav Pediatr 27(3):188-192

38. Jain BP, Pandey S (2018) WD40 repeat proteins: Signalling scaffold with diverse functions. Protein J 37(5):391-406

39. Stein JL, Hibar DP, Madsen SK, Khamis M, McMahon KL, de Zubicaray Gl et al (2011) Discovery and replication of dopamine-related gene effects on caudate volume in young and elderly populations ( $\mathrm{N}=1198)$ using genomewide search. Mol Psychiatry 16(9):927-937 881

40. Zhang D, lyer LM, He F, Aravind L (2012) Discovery of novel DENN proteins: implications for the evolution of eukaryotic intracellular membrane structures and human disease. Front Genet 3:283

41. Levine TP, Daniels RD, Gatta AT, Wong LH, Hayes MJ (2013) The product of C9orf72, a gene strongly implicated in neurodegeneration, is structurally related to DENN Rab-GEFs. Bioinformatics. 29(4):499-503

42. Zhen Y, Stenmark H (2015) Cellular functions of Rab GTPases at a glance. J Cell Sci 128(17):3171-3176

43. Farg MA, Sundaramoorthy $V$, Sultana JM, Yang S, Atkinson RA, Levina $V$ et al (2014) C9ORF72, implicated in amytrophic lateral sclerosis and frontotemporal dementia, regulates endosomal trafficking. Hum Mol Genet 23(13):3579-3595

44. Webster CP, Smith EF, Grierson AJ, De Vos KJ (2018) C9orf72 plays a central role in Rab GTPase-dependent regulation of autophagy. Small GTPases. 9(5):399-408

45. O'Rourke JG, Bogdanik L, Yanez A, Lall D, Wolf AJ, Muhammad AK et al (2016) C9orf72 is required for proper macrophage and microglial function in mice. Science 351(6279):1324-1329

46. Aoki Y, Manzano R, Lee Y, Dafinca R, Aoki M, Douglas AGL et al (2017) C9orf72 and RAB7L1 regulate vesicle trafficking in amyotrophic lateral sclerosis and frontotemporal dementia. Brain 140(4):887-897

47. Leskela S, Huber N, Rostalski H, Natunen T, Remes AM, Takalo M et al (2019) C9orf72 proteins regulate autophagy and undergo Autophagosomal or proteasomal degradation in a cell type-dependent manner. Cells 8(10):1233

48. Lai JD, Ichida JK (2019) C9ORF72 protein function and immune dysregulation in amyotrophic lateral sclerosis. Neurosci Lett 713:134523

49. Corrionero A, Horvitz HR (2018) A C9orf72 ALS/FTD ortholog acts in endolysosomal degradation and lysosomal homeostasis. Curr Biol 28(10): 1522-35.e5

50. Amick J, Tharkeshwar AK, Talaia G, Ferguson SM (2020) PQLC2 recruits the C9orf72 complex to lysosomes in response to cationic amino acid starvation. J Cell Biol 219(1):e201906076

51. Sivadasan R, Hornburg D, Drepper C, Frank N, Jablonka S, Hansel A et al (2016) C9ORF72 interaction with cofilin modulates actin dynamics in motor neurons. Nat Neurosci 19(12):1610-1618

52. Selvaraj BT, Livesey MR, Zhao C, Gregory JM, James OT, Cleary EM et al (2018) C9ORF72 repeat expansion causes vulnerability of motor neurons to $\mathrm{Ca}(2+)$-permeable AMPA receptor-mediated excitotoxicity. Nat Commun 9(1):347

53. Staats KA, Seah C, Sahimi A, Wang Y, Koutsodendris N, Lin S, et al. Small molecule inhibition of PIKFYVE kinase rescues gain- and loss-of-function
C9ORF72 ALS/FTD disease processes in vivo. bioRxiv. 2019. https://doi.org/ $10.1101 / 685800$

54. Xiao S, McKeever PM, Lau A, Robertson J (2019) Synaptic localization of C9orf72 regulates post-synaptic glutamate receptor 1 levels. Acta Neuropathol Commun. 7(1):161

55. Ciura S, Lattante S, Le Ber I, Latouche M, Tostivint H, Brice A et al (2013) Loss of function of C9orf72 causes motor deficits in a zebrafish model of amyotrophic lateral sclerosis. Ann Neurol 74(2):180-187

56. Therrien M, Rouleau GA, Dion PA, Parker JA (2013) Deletion of C9ORF72 results in motor neuron degeneration and stress sensitivity in C. elegans. PLoS One 8(12):e83450

57. Shao Q, Yang M, Liang C, Ma L, Zhang W, Jiang Z et al (2019) C9orf72 and Smcr8 mutant mice reveal MTORC1 activation due to impaired lysosomal degradation and exocytosis. Autophagy:1-16

58. Lagier-Tourenne C, Baughn M, Rigo F, Sun S, Liu P, Li H-R et al (2013) Targeted degradation of sense and antisense C9orf72 RNA foci as therapy for ALS and frontotemporal degeneration. Proc Natl Acad Sci U S A 110(47): E4530-E4539

59. Koppers M, Blokhuis AM, Westeneng HJ, Terpstra ML, Zundel CA (2015) Vieira de Sa R, et al. C9orf72 ablation in mice does not cause motor neuron degeneration or motor deficits. Ann Neurol 78(3):426-438

60. Atanasio A, Decman V, White D, Ramos M, Ikiz B, Lee HC et al (2016) C9orf72 ablation causes immune dysregulation characterized by leukocyte expansion, autoantibody production, and glomerulonephropathy in mice. Sci Rep 6:23204

61. Burberry A, Suzuki N, Wang JY, Moccia R, Mordes DA, Stewart MH et al (2016) Loss-of-function mutations in the C9ORF72 mouse ortholog cause fatal autoimmune disease. Sci Transl Med 8(347):347ra93

62. Sudria-Lopez E, Koppers M, de Wit M, van der Meer C, Westeneng HJ, Zundel CA et al (2016) Full ablation of C9orf72 in mice causes immune system-related pathology and neoplastic events but no motor neuron defects. Acta Neuropathol 132(1):145-147

63. Jiang J, Zhu Q, Gendron TF, Saberi S, McAlonis-Downes M, Seelman A et al (2016) Gain of toxicity from ALS/FTD-linked repeat expansions in C9ORF72 is alleviated by antisense oligonucleotides targeting GGGGCC-containing RNAs. Neuron 90(3):535-550

64. McAlpine W, Sun L, Wang KW, Liu A, Jain R, San Miguel M et al (2018) Excessive endosomal TLR signaling causes inflammatory disease in mice with defective SMCR8-WDR41-C9ORF72 complex function. Proc Natl Acad Sci U S A 115(49):E11523-E11e31

65. Zhang Y, Burberry A, Wang JY, Sandoe J, Ghosh S, Udeshi ND et al (2018) The C9orf72-interacting protein Smcr8 is a negative regulator of autoimmunity and lysosomal exocytosis. Genes Dev 32(13-14):929-943

66. Harms MB, Cady J, Zaidman C, Cooper P, Bali T, Allred P et al (2013) Lack of C9ORF72 coding mutations supports a gain of function for repeat expansions in amyotrophic lateral sclerosis. Neurobiol Aging 34(9):2234.e13-2234.e19

67. Henderson MJ, Russell AJ, Hird S, Munoz M, Clancy JL, Lehrbach GM et al (2002) EDD, the human hyperplastic discs protein, has a role in progesterone receptor coactivation and potential involvement in DNA damage response. J Biol Chem 277(29):26468-26478

68. Zhang YJ, Gendron TF, Grima JC, Sasaguri H, Jansen-West K, Xu YF et al (2016) C9ORF72 poly(GA) aggregates sequester and impair HR23 and nucleocytoplasmic transport proteins. Nat Neurosci 19(5):668-677

69. Zhang T, Baldie G, Periz G, Wang J (2014) RNA-processing protein TDP-43 regulates $\mathrm{FOXO}$-dependent protein quality control in stress response. PLoS Genet 10(10):e1004693

70. Bergink S, Salomons FA, Hoogstraten D, Groothuis TA, de Waard H, Wu J et al (2006) DNA damage triggers nucleotide excision repair-dependent monoubiquitylation of histone H2A. Genes Dev 20(10):1343-1352

71. Ko HS, Bailey R, Smith WW, Liu Z, Shin JH, Lee Yl et al (2009) CHIP regulates leucine-rich repeat kinase-2 ubiquitination, degradation, and toxicity. Proc Natl Acad Sci U S A 106(8):2897-2902

72. Agrawal $P$, Chen YT, Schilling B, Gibson BW, Hughes RE (2012) Ubiquitinspecific peptidase 9, X-linked (USP9X) modulates activity of mammalian target of rapamycin (mTOR). J Biol Chem 287(25):21164-21175

73. Hulme AE, Bogerd HP, Cullen BR, Moran JV (2007) Selective inhibition of Alu retrotransposition by APOBEC3G. Gene. 390(1):199-205

74. Batra R, Hutt K, Vu A, Rabin SJ, Baughn MW, Libby RT et al (2016) Gene expression signatures of sporadic ALS motor neuron populations. bioRxiv. https://doi.org/10.1101/038448 
75. Goodier JL, Cheung LE, Kazazian HH (2013) Mapping the LINE1 ORF1 protein interactome reveals associated inhibitors of human retrotransposition. Nucleic Acids Res 41(15):7401-7419

76. Goodier JL, Pereira GC, Cheung LE, Rose RJ, Kazazian HH (2015) The broadspectrum antiviral protein ZAP restricts human retrotransposition. PLoS Genet 11(5):e1005252

77. Stoecklin G, Mayo T, Anderson P (2006) ARE-mRNA degradation requires the 5'-3' decay pathway. EMBO Rep 7(1):72-77

78. Rodić N, Sharma R, Sharma R, Zampella J, Dai L, Taylor MS et al (2014) Long interspersed element-1 protein expression is a hallmark of many human cancers. Am J Pathol 184(5):1280-1286

79. Laflamme C, McKeever PM, Kumar R, Schwartz J, Kolahdouzan M, Chen CX et al (2019) Implementation of an antibody characterization procedure and application to the major ALS/FTD disease gene C9ORF72. eLife 8:e48363

80. Eystathioy T, Chan EK, Takeuchi K, Mahler M, Luft LM, Zochodne DW et a (2003) Clinical and serological associations of autoantibodies to GW bodies and a novel cytoplasmic autoantigen GW182. J Mol Med (Berl) 81(12):811-818

81. Goodier JL, Zhang L, Vetter MR, Kazazian HH (2007) LINE-1 ORF1 protein localizes in stress granules with other RNA-binding proteins, including components of RNA interference RNA-induced silencing complex. Mol Cell Biol 27(18):6469-6483

82. Goodier JL, Mandal PK, Zhang L, Kazazian HH (2010) Discrete subcellular partitioning of human retrotransposon RNAs despite a common mechanism of genome insertion. Hum Mol Genet 19(9):1712-1725

83. Huang da W, Sherman BT, Lempicki RA (2009) Systematic and integrative analysis of large gene lists using DAVID bioinformatics resources. Nat Protoc 4(1):44-57

84. Jin Y, Tam OH, Paniagua E, Hammell M (2015) TEtranscripts: a package for including transposable elements in differential expression analysis of RNAseq datasets. Bioinformatics. 31(22):3593-3599

85. Pereira GC, Sanchez L, Schaughency PM, Rubio-Roldan A, Choi JA, Planet E et al (2018) Properties of LINE-1 proteins and repeat element expression in the context of amyotrophic lateral sclerosis. Mob DNA 9:35

86. Radivojac P, Vacic V, Haynes C, Cocklin RR, Mohan A, Heyen JW et al (2010) Identification, analysis, and prediction of protein ubiquitination sites. Proteins. 78(2):365-380

87. Blokhuis AM, Koppers M, Groen EJN, van den Heuvel DMA, Dini Modigliani S, Anink JJ et al (2016) Comparative interactomics analysis of different ALS associated proteins identifies converging molecular pathways. Acta Neuropathol 132(2):175-196

88. Chitiprolu M, Jagow C, Tremblay V, Bondy-Chorney E, Paris G, Savard A et al (2018) A complex of C9ORF72 and p62 uses arginine methylation to eliminate stress granules by autophagy. Nat Commun 9(1):2794

89. Tang D, Sheng J, Xu L, Zhan X, Liu J, Jiang H et al (2020) Cryo-EM structure of C9ORF72-SMCR8-WDR41 reveals the role as a GAP for Rab8a and Rab11a. Proc Natl Acad Sci U S A 117(18):9876-9883

90. Kopito RR (2000) Aggresomes, inclusion bodies and protein aggregation. Trends Cell Biol 10(12):524-530

91. Gamerdinger M, Kaya AM, Wolfrum U, Clement AM, Behl C (2011) BAG3 mediates chaperone-based aggresome-targeting and selective autophagy of misfolded proteins. EMBO Rep 12(2):149-156

92. Zhang X, Qian SB (2011) Chaperone-mediated hierarchical control in targeting misfolded proteins to aggresomes. Mol Biol Cell 22(18):3277-3288

93. Bentmann E, Haass C, Dormann D (2013) Stress granules in neurodegeneration--lessons learnt from TAR DNA binding protein of $43 \mathrm{kDa}$ and fused in sarcoma. FEBS J 280(18):4348-4370

94. Fernandes N, Eshleman N, Buchan JR (2018) Stress granules and ALS: a case of causation or correlation? Adv Neurobiol 20:173-212

95. Baradaran-Heravi Y, Van Broeckhoven C, van der Zee J (2020) Stress granule mediated protein aggregation and underlying gene defects in the FTD-ALS spectrum. Neurobiol Dis 134:104639

96. Anderson P, Kedersha N (2006) RNA granules. J Cell Biol 172(6):803-808

97. Kwon S, Zhang Y, Matthias P (2007) The deacetylase HDAC6 is a novel critical component of stress granules involved in the stress response. Genes Dev 21(24):3381-3394

98. Maharjan N, Kunzli C, Buthey K, Saxena S (2017) C9ORF72 regulates stress granule formation and its deficiency impairs stress granule assembly, hypersensitizing cells to stress. Mol Neurobiol 54(4):3062-3077

99. Souquere S, Mollet S, Kress M, Dautry F, Pierron G, Weil D (2009) Unravelling the ultrastructure of stress granules and associated P-bodies in human cells. J Cell Sci 122(Pt 20):3619-3626

100. Kawakami I, Arai T, Hasegawa M (2019) The basis of clinicopathological heterogeneity in TDP-43 proteinopathy. Acta Neuropathol 138(5):751-770
101. Freibaum BD, Taylor JP (2017) The role of dipeptide repeats in C9ORF72 related ALS-FTD. Front Mol Neurosci 10:35

102. Nunes C, Mestre I, Marcelo A, Koppenol R, Matos CA, Nobrega C (2019) MSGP: the first database of the protein components of the mammalian stress granules. Database (Oxford) 2019:baz031

103. Lan Y, Sullivan PM, Hu F (2019) SMCR8 negatively regulates AKT and MTORC1 signaling to modulate lysosome biogenesis and tissue homeostasis. Autophagy 15(5):871-885

104. Schneider CA, Rasband WS, Eliceiri KW (2012) NIH image to ImageJ: 25 years of image analysis. Nat Methods 9(7):671-675

105. Grumati P, Dikic I (2018) Ubiquitin signaling and autophagy. J Biol Chem 293(15):5404-5413

106. Chen RH, Chen YH, Huang TY (2019) Ubiquitin-mediated regulation of autophagy. J Biomed Sci 26(1):80

107. Rao L, Sha Y, Eissa NT (2017) The E3 ubiquitin ligase STUB1 regulates autophagy and mitochondrial biogenesis by modulating TFEB activity. Mol Cell Oncol 4(6):e1372867

108. Wan W, You Z, Zhou L, Xu Y, Peng C, Zhou T et al (2018) mTORC1regulated and HUWE1-mediated WIPI2 degradation controls autophagy flux. Mol Cell 72(2):303-15.e6

109. Amick J, Ferguson SM (2017) C9orf72: at the intersection of lysosome cell biology and neurodegenerative disease. Traffic 18(5):267-276

110. Jung J, Behrends C (2020) Multifaceted role of SMCR8 as autophagy regulator. Small GTPases 11(1):53-61

111. Mazroui R, Di Marco S, Kaufman R, Gallouzi IE (2007) Inhibition of the ubiquitinproteasome system induces stress granule formation. Mol Biol Cell 18(7):2603-2618

112. Mateju D, Franzmann TM, Patel A, Kopach A, Boczek EE, Maharana S et al (2017) An aberrant phase transition of stress granules triggered by misfolded protein and prevented by chaperone function. EMBO J 36(12):1669-1687

113. Xie X, Matsumoto S, Endo A, Fukushima T, Kawahara H, Saeki Y et al (2018) Deubiquitylases USP5 and USP13 are recruited to and regulate heat-induced stress granules through their deubiquitylating activities. J Cell Sci 131(8):jcs210856

114. Seguin SJ, Morelli FF, Vinet J, Amore D, De Biasi S, Poletti A et al (2014) Inhibition of autophagy, lysosome and VCP function impairs stress granule assembly. Cell Death Differ 21(12):1838-1851

115. Monahan Z, Shewmaker F, Pandey UB (2016) Stress granules at the intersection of autophagy and ALS. Brain Res 1649(Pt B):189-200

116. Vu LT, Bowser R (2017) Fluid-based biomarkers for amyotrophic lateral sclerosis. Neurotherapeutics. 14(1):119-134

117. van der Ende EL, Meeter LH, Stingl C, van Rooij JGJ, Stoop MP, Nijholt DAT et al (2019) Novel CSF biomarkers in genetic frontotemporal dementia identified by proteomics. Ann Clin Transl Neurol 6(4):698-707

118. Beck J, Poulter M, Hensman D, Rohrer JD, Mahoney CJ, Adamson G et al (2013) Large C9orf72 hexanucleotide repeat expansions are seen in multiple neurodegenerative syndromes and are more frequent than expected in the UK population. Am J Hum Genet 92(3):345-353

119. Hensman Moss DJ, Poulter M, Beck J, Hehir J, Polke JM, Campbell T et al (2014) C9orf72 expansions are the most common genetic cause of Huntington disease phenocopies. Neurology. 82(4):292-299

120. Bourinaris T, Houlden $H$ (2018) C9orf72 and its relevance in parkinsonism and movement disorders: a comprehensive review of the literature. Mov Disord Clin Pract 5(6):575-585

121. Devenney EM, Ahmed RM, Halliday G, Piguet O, Kiernan MC, Hodges JR (2018) Psychiatric disorders in C9orf72 kindreds: study of 1,414 family members. Neurology 91(16):e1498-ee507

122. Silverman HE, Goldman JS, Huey ED (2019) Links between the C9orf72 repeat expansion and psychiatric symptoms. Curr Neurol Neurosci Rep 19(12):93

123. Marogianni C, Rikos D, Provatas A, Dadouli K, Ntellas P, Tsitsi P et al (2019) The role of C9orf72 in neurodegenerative disorders: a systematic review, an updated meta-analysis, and the creation of an online database. Neurobiol Aging 84:238.e25-238.e34

\section{Publisher's Note}

Springer Nature remains neutral with regard to jurisdictional claims in published maps and institutional affiliations. 